\title{
Identification Properties of Recent Production Function Estimators
}

\author{
Daniel A. Ackerberg, Kevin Caves, and Garth Frazer*
}

July 14, 2015

\begin{abstract}
This paper examines some of the recent literature on the estimation of production functions. We focus on techniques suggested in two recent papers, Olley and Pakes (1996), and Levinsohn and Petrin (2003). While there are some solid and intuitive identification ideas in these papers, we argue that the techniques can suffer from functional dependence problems. We suggest an alternative approach that is based on the ideas in these papers, but does not suffer from the functional dependence problems and produces consistent estimates under alternative data generating processes for which the original procedures do not.
\end{abstract}

\section{Introduction}

Production functions are a fundamental component of all economics. As such, estimation of production functions has a long history in applied economics, starting in the early 1800's. Unfortunately, this history cannot be deemed an unqualified success, as many of the econometric problems hampering early estimation are still an issue today.

Production functions relate output to inputs (e.g. capital, labor). Perhaps the major econometric issue in estimating production functions is the possibility that there are determinants of production that are unobserved to the econometrician but observed by the firm. If this is the case, and if the observed inputs are chosen as a function of these determinants (as will typically be the case for a profit-maximizing or cost-minimizing firm), then there is an endogeneity problem and OLS estimates of the coefficients on the observed inputs will be biased. The literature has devoted much attention to the problem of these biases and approaches to solving them (see, e.g. Marschak and Andrews (1944), Hoch (1955, 1958, 1962), Mundlak (1961, 1963, 1996), Mundlak and Hoch (1965), Christensen, Jorgenson, and Lau (1971), Christensen and Greene (1976), McElroy (1987), Panzar (1989), and Slade (1989).)

Over the past 20 years, techniques proposed by Olley and Pakes (1996) (OP) and Levinsohn and Petrin (2003) (LP) to address this endogeneity problem have seen extensive use in the empirical literature, e.g. Pavcnik (2002), Criscuola and Martin (2003), Fernandes (2003), Blalock and Gertler (2004), Alvarez and Lopez (2005), Sivadasan (2009), Ozler and Yilmaz (2009), Topalova and Khandewal (2011), and numerous others. The essence of the OP and LP techniques is that, under certain theoretical and statistical assumptions, one can invert optimal input decisions to essentially allow an econometrician to "observe" unobserved productivity shocks. More precisely, OP identify conditions under which firm level investment (conditional on capital stock) is a strictly increasing function of a scalar, firm-level, unobserved productivity shock. This strict monotonicity implies that one can invert this investment demand function, and thus "control for" the unobserved productivity shock by conditioning on a nonparametric representation of that inverse function (i.e. a non-parametric function of capital stock and investment).

*Dept. of Economics, University of Michigan; Economists Inc., Washington D.C.; and Department of Management - UTSC, Rotman School of Management, and School of Public Policy and Governance, University of Toronto respectively. Earlier versions of this paper circulated under the title "Structural Identification of Production Functions". Thanks to two co-editors, three referees, and Lanier Benkard, Uli Doraszelski, Jeremy Fox, Amit Gandhi, Gautam Gowrisankaran, Jinyong Hahn, Ariel Pakes, Amil Petrin, Mike Riordan and seminar participants at UCLA, USC, UCSD, NYU, Columbia, Iowa State, Toronto, ESWC-2005, Yale, Northwestern, Colorado, Indiana, Oregon State, Nice, and Stanford for helpful discussions. All errors are our own. 
In the first stage of the approach, one estimates the labor coefficient in the production function by regressing output on the labor input and this non-parametric function. LP use a similar approach, but invert an intermediate input demand function instead of an investment demand function to control for the unobserved productivity shock.

This paper first argues that there may be a problem with the first stage of these estimation procedures. More specifically, under some simple data generating processes that are consistent with the stated assumptions of the OP/LP models, the moment condition underlying the first stage estimating equation does not identifiy the labor coefficient. The problem is one of functional dependence. Under these data generating process, one can show that labor is a deterministic function of the set of variables that, in the OP/LP procedures, need to be non-parametrically conditioned on. Hence, once one does this non-parametric conditioning, there is no variation in labor left to identify the labor coefficient. ${ }^{1}$ We then explore alternative data generating processes, trying to find examples where this functional dependence problem does not apply. We find only a limited set of data generating processes for which the labor coefficient can be identified using the OP/LP first stage moments, characterize these processes, and argue that they may be unrealistic in many industries.

We then propose an alternative estimation procedure that uses moment conditions very similar to those used by OP and LP, but that avoid this functional dependence problem. Specifically, while OP and LP invert investment (OP) and intermediate input (LP) demand functions that are unconditional on the labor input, we suggest inverting investment or intermediate demand functions that are conditional on the labor input. While use of these conditional input demand functions precludes identification of the labor coefficient using the first stage moments, we describe how this coefficient can be estimated along with the other production function parameters using the second stage moments (after the first stage moments are used to estimate auxiliary parameters). We also illustrate how our use of conditional input demand functions allows for some more general data generating processes than the original OP and LP procedures. Unlike the canonical OP and LP moment conditions, our moment conditions produce consistent estimates if there are unobserved, serially correlated, firm-specific shocks to the price of labor, if labor is chosen prior to other variable inputs (with a different information set), or if labor is dynamic and there are unobserved, firm-specific adjustment costs of labor. Wooldridge (2009) proposes estimating the two sets of moments in LP (or OP) simultaneously, and notes that doing this avoids the same functional dependence issue raised in a working paper version of this paper. On the other hand, as we discuss in Section 4.3.2, the Wooldridge moments as written are based on unconditional input demand functions and do not allow all the aforementioned data generating processes.

We start with a quick review of the various approaches that have been used to address this endogeneity problem over the past 70 years. We then discuss the potential functional dependence issues in OP and LP and describe the data generating processes necessary to avoid these functional dependence issues. We then present our alternative moments and estimation procedure and discuss in what dimensions these alternative moments extend the data generating processes that can be considered. Lastly, we perform a brief Monte-Carlo study. We consider some data generating processes that are favorable to the LP moments, and some that are favorable to our alternative moments, and as expected, each procedure performs better in the respective data generating process that is favorable to it. Perhaps more interestingly, we then create misspecification by adding measurement error to the material input variable. Neither our moments nor the LP moments produce consistent estimates, but estimates from our moments are less sensitive than the LP moments to at least this form of misspecification.

\section{Review of Literature on Production Function Estimation}

We start with a brief review of the literature on estimation of production functions (borrowing liberally from Griliches and Mairesse (1998)), ending with a detailed summary of the approaches of Olley and Pakes (1996, henceforth OP) and Levinsohn and Petrin (2003, henceforth LP). OP and LP are the approaches which our work

\footnotetext{
${ }^{1}$ Susanto Basu made a less formal argument regarding this possible functional dependence problem as a discussant of an earlier version of the Levinsohn-Petrin paper.
} 
most directly assesses and builds on.

For illustration purposes, consider a simple Cobb-Douglas production function in logs

$$
y_{i t}=\beta_{0}+\beta_{k} k_{i t}+\beta_{l} l_{i t}+\omega_{i t}+\epsilon_{i t}
$$

where $y_{i t}$ is the $\log$ of output, $k_{i t}$ is the $\log$ of capital input, and $l_{i t}$ is the log of labor input, all of which are observed. There are two econometric unobservables, $\omega_{i t}$ and $\epsilon_{i t}$. The $\epsilon_{i t}$ represent shocks to production or productivity that are not observable (or predictable) by firms before making their input decisions at $t$. In contrast, the $\omega_{i t}$ represent "productivity" shocks that are potentially observed or predictable by firms when they make input decisions. Intuitively, $\omega_{i t}$ might represent variables such as the managerial ability of a firm, expected down-time due to machine breakdown, expected defect rates in a manufacturing process, soil quality, or the expected rainfall at a particular farm's location. $\epsilon_{i t}$ might represent deviations from expected breakdown, defect, or rainfall amounts in a given year. $\epsilon_{i t}$ can also represent (potentially serially correlated) measurement error in the output variable.

The crux of the identification problem inherent in estimating such a production function is that the inputs $k_{i t}$ and $l_{i t}$ are chosen by firms. This means that if the econometric unobservable $\omega_{i t}$ is observed (or partially observed) by the firm prior to choosing $k_{i t}$ and $l_{i t}$, then these choices will likely depend on $\omega_{i t}$, creating correlation between $\left(k_{i t}, l_{i t}\right)$ and $\omega_{i t}$, and rendering OLS estimates of $\beta_{k}$ and $\beta_{l}$ inconsistent. Observation of this endogeneity problem dates back to Marschak and Andrews (1944).

\subsection{Prior Approaches}

One approach to address this endogeneity problem involved using panel data with fixed effects, first advocated by Hoch (1955, 1962), Mundlak (1961, 1963), and Mundlak and Hoch (1965). The most basic of these approaches involves assuming $\omega_{i t}=\omega_{i}$, where the "fixed effect" $\omega_{i}$ is observed by the firm prior to choosing inputs. In essence the assumption here is that all endogeneity problems are related to a time-invariant shock $\omega_{i}$. Under this assumption standard fixed effect approaches can consistently estimate $\beta_{k}$ and $\beta_{l}$. Unfortunately, this approach has not worked well in practice - one common finding is unreasonably low estimates of $\beta_{k}$ and returns to scale. As noted by Griliches and Mairesse (1998), this could be due to problems with the assumption that the only unobservable generating endogeneity problems is time invariant, or it could be due to problems with data (since differencing can exacerbate measurement error problems, e.g. Griliches and Hausman (1986)). In recent years, these approaches have been generalized (e.g. Chamberlain (1982), Anderson and Hsiao (1982), Arellano and Bond (1991), Arellano and Bover (1995), and Blundell and Bond $(1998,2000)$ ) - see section 4.3.3.

A second approach has utilized information in firms' first order conditions with respect to inputs. Early applications include Klein (1953), Solow (1957), Nerlove (1963), Griliches and Ringstad (1971), and Hall (1988). Generally speaking, these approaches consider estimation of first order conditions regarding, e.g. choices of $k_{i t}$ and $l_{i t}$. These first order conditions depend on input (and output) prices, as well as the production function parameters (the simplest illustration of this approach is the well known observation that in a Cobb-Douglas environment, the first order conditions imply that the cost shares of each input equal the respective input elasticities). These approaches, including the related idea of estimating cost functions (e.g. Christensen, Jorgenson, and Lau (1971), Christensen and Greene (1976)), have been generalized in a number of dimensions, including some fairly rich theoretical and statistical models. A good example is McElroy (1987), who utilizes first order conditions in the context of a production function more general than (1). McElroy not only considers a more flexible production function (translog), but also a more flexible stochastic specification in which there are multiple structural unobservables entering in different places in the production function (instead of only Hicks neutral shocks as in (1)) - specifically, there is one such structural unobservable associated with each of the inputs, so, e.g., some firms are relatively more efficient at using capital, and some are relatively more efficient at using labor. One caveat of these approaches is that they typically rely on the assumption that input choices satisfy static first order conditions. This could be 
problematic if certain inputs were subject to significant dynamic issues, e.g. adjustment costs, or wedges between purchase and resale prices. A particular worry with respect to this caveat might be the capital input, which in many theoretical models has dynamic implications so that the static first order condition does not hold. In theory one could construct first order conditions consistent with these dynamic implications, but these would 1) be considerably more complicated than static first order conditions, and 2) likely require additional auxiliary assumptions on the dynamic environment firms operate in.

As pointed out by Griliches and Mairesse (1998), a natural extension of the above "first order condition" approaches involves using input prices as "instruments" for the observed inputs in (1), i.e. by simply regressing $y_{i t}$ on $k_{i t}$ and $l_{i t}$, using $p_{i t}^{k}$ and $p_{i t}^{l}$ as instruments. The idea here is that if input prices are exogenous (i.e. uncorrelated with $\omega_{i t}+\epsilon_{i t}$ ), and if input choices respond to these input prices (which they should), then these input prices provide a source of exogenous variation in $k_{i t}$ and $l_{i t}$ with which to identify $\beta_{k}$ and $\beta_{l}$. What is particularly nice about this simple "input price based IV" method is that its consistency does not require one to specify the relationship between the inputs $\left(k_{i t}\right.$ and $l_{i t}$ ), and input prices using $p_{i t}^{k}$ and $p_{i t}^{l}$ correctly. Hence, unlike the aforementioned work that relies on using static first order conditions explicitly, it can produce consistent estimates even if the inputs are dynamic in nature (though it will generally not be as efficient if the static first order conditions do hold). Of course, these "input price based IV" methods rely on the researcher observing variation in input prices across firms, and the assumption that these input prices are exogenous. Griliches and Mairesse (1998) note a number of reasons to potentially question this assumption. For it to hold, e.g., the observed price differences across firms cannot capture input quality differences across firms, or different firms' choices of location on a downward sloping input supply curve.

Much of the motivation behind the OP (and LP) approaches is related to the caveats described above. For example, unlike pure fixed effect approaches, OP and LP do allow for input endogeneity with respect to a time varying unobservable. Unlike many of the first order condition approaches, they can allow for subsets of inputs to be dynamic in nature, yet do not require explicit solution of complicated dynamic first order conditions related to those inputs. And lastly, unlike the "input price based IV" methods, they do not require the econometrician to observe exogeneous, across-firm variation in input prices.

Of course, relaxing these assumptions does not come without costs. One needs a new set of statistical and theoretical restrictions to produce identification in the OP and LP models. As detailed extensively below, potentially strong other assumptions need to be made regarding, e.g. similarities in the environments that different firms face, what variables are in firms' information sets when different inputs are chosen, and limiting the amount of unobserved heterogeneity in production functions across firms. We see this paper as contributing to the literature by 1) clarifying these statistical and theoretical restrictions, 2) pointing out some significant caveats of these statistical and theoretical restrictions related to functional dependence issues, and 3) proposing some alternative (but very similar) estimators that avoid these functional dependence problems. It is important to point out that while we relax a few of the assumptions of OP/LP and avoid the functional dependence issue, the key assumptions behind our approach remain the same. Our approach, like OP and LP, relies critically on timing assumptions, a scalar unobservable assumption, and a monotonicity assumption. These potentially strong assumptions are what allow for the relaxed assumptions in other dimensions described above. Naturally, whether one chooses our approach or that of the other estimation methods, one should strive for transparency regarding key assumptions and then motivate the appropriateness of those assumptions for the relevant empirical context.

\subsection{Olley/Pakes and Levinsohn/Petrin}

Behind the OP and LP identification strategy is a discrete time model of dynamically optimizing firms. Typically the primitives of the model are assumed to satisfy the following three assumptions:

Assumption 1 Assumption 1: (Information Set) The firm's information set at $t$, i.e. $I_{i t}$, includes current and 
past productivity shocks $\left\{\omega_{i \tau}\right\}_{\tau=0}^{t}$ but does not include future productivity shocks $\left\{\omega_{i \tau}\right\}_{\tau=t+1}^{\infty}$. The transitory shocks $\epsilon_{i t}$ satisfy $E\left[\epsilon_{i t} \mid I_{i t}\right]=0$.

Assumption 2 Assumption 2: (First Order Markov) Productivity shocks evolve according to the distribution

$$
p\left(\omega_{i t+1} \mid I_{i t}\right)=p\left(\omega_{i t+1} \mid \omega_{i t}\right)
$$

This distribution is known to firms and stochastically increasing in $\omega_{i t}$.

Assumption 3 Assumption 3: (Timing of Input Choices) Firms accumulate capital according to

$$
k_{i t}=\kappa\left(k_{i t-1}, i_{i t-1}\right)
$$

where investment $i_{i t-1}$ is chosen in period $t-1$. Labor input $l_{i t}$ is non-dynamic and chosen at $t$.

Assumptions (1) and (2) are straightforward - firms do not observe $\omega_{i t}$ until time $t$, but the distribution $p\left(\omega_{i t+1} \mid \omega_{i t}\right)$ defines what the firm knows about the distribution of future productivity shocks. Assumption (3) distinguishes the capital and labor inputs. The labor input is non-dynamic in the sense that a firm's choice of labor for period $t$ has no impact on the future profits of the firm. In contrast, capital is assumed to be a dynamic input subject to an investment process. Importantly, Assumption (3) implies that the period $t$ capital stock of the firm was actually determined at period $t-1$, i.e. $k_{i t} \in I_{i t-1}$. Intuitively, the restriction behind this assumption is that it takes a full period for new capital to be ordered, delivered, and installed. This assumption will be important in generating the moment conditions used for estimation. Neither OP nor LP precisely define the data-generating process (DGP) determining $l_{i t}$. One of the contributions of this paper is to think about this DGP more formally, which we do in Section 3.

In the above model, firms' optimal investment decisions $i_{i t}$ are policy functions resulting from a dynamic optimization problem. Under additional assumptions on primitives of the model, OP derive two crucial properties of these policy functions. To save space, we simply state these as additional, high-level, assumptions

Assumption 4 Assumption 4: (Scalar Unobservable) Firms'investment decisions are given by

$$
i_{i t}=f_{t}\left(k_{i t}, \omega_{i t}\right)
$$

Assumption 5 Assumption 5: (Strict Monotonicity) $f_{t}\left(k_{i t}, \omega_{i t}\right)$ is strictly increasing in $\omega_{i t}$.

Assumption (4) states that investment is a function of the state variables $k_{i t}$ and $\omega_{i t}\left(l_{i t}\right.$ is not a state variable because it is non-dynamic and chosen at $t$ ). Griliches and Mairesse (1998) note that Assumption (4) places strong implicit restrictions on additional firm-specific econometric unobservables in the model. For example, it rules out any unobserved heterogeneity across firms in adjustment costs of capital, in demand or labor market conditions (unless they evolve independently over time), or additional unobservables entering other parts of the production function as in McElroy (1987). On the other hand, the indexing of $f$ by $t$ does allow differences in these variables 
across time. $^{2}$ Assumption (5) is a consequence of the assumptions that $p\left(\omega_{i t+1} \mid \omega_{i t}\right)$ is stochastically increasing in $\omega_{i t}$ and that $\omega_{i t}$ positively affects the marginal product of capital - together these imply that firms with higher $\omega_{i t}$ 's have higher expected marginal products of capital in the future, and thus engage in more investment. Formally proving this requires using dynamic programming methods and can be a challenge in more complicated models. Mechanically speaking, all the results here and in later sections can apply to production functions more general than Cobb-Douglas given that Assumptions 1 through 5 hold (so, e.g., they cannot be applied to production functions with multidimensional unobservables like McElroy (1987)).

The econometrician is presumed to observe inputs $l_{i t}$ and $k_{i t}$, output $y_{i t}$, and investment levels $i_{i t}$ for a panel dataset of firms with $N \rightarrow \infty$ and fixed $T$. We assume that inputs and outputs are measured in comparable physical units across observations (extensions to this are discussed in Section 4.3.4.) Estimation in OP proceeds in two stages, each stage corresponding to a distinct moment condition. To form the "first stage" moment condition, note that Assumptions (4) and (5) imply that one can invert the investment policy function

$$
\omega_{i t}=f_{t}^{-1}\left(k_{i t}, i_{i t}\right)
$$

to write the productivity shock as a (unknown) function of observables. Substituting this into the production function, we get:

$$
y_{i t}=\beta_{0}+\beta_{k} k_{i t}+\beta_{l} l_{i t}+f_{t}^{-1}\left(k_{i t}, i_{i t}\right)+\epsilon_{i t}=\beta_{l} l_{i t}+\Phi_{t}\left(k_{i t}, i_{i t}\right)+\epsilon_{i t}
$$

Because $f_{t}^{-1}$ is the solution to a potentially complicated dynamic programming problem, deriving its functional form would require additional computation and specifying additional primitives (e.g. the evolution of industrywide input prices or demand conditions over time). To avoid this, OP treat $f_{t}^{-1}$ non-parametrically. This clearly precludes identification of $\beta_{0}$ and $\beta_{k}$ using this equation, so these are combined into a composite term $\Phi_{t}\left(k_{i t}, i_{i t}\right)$ that is treated non-parametrically.

The first stage of OP then generates GMM estimates $\widehat{\beta}_{l}$ and $\widehat{\Phi}_{t}\left(k_{i t}, i_{i t}\right)$ using the moment condition

$$
E\left[\epsilon_{i t} \mid I_{i t}\right]=E\left[y_{i t}-\beta_{l} l_{i t}-\Phi_{t}\left(k_{i t}, i_{i t}\right) \mid I_{i t}\right]=0
$$

If one uses a polynomial approximation to $\Phi_{t}$, this can be as simple as running OLS of $y_{i t}$ on $l_{i t}$ and the polynomial.

For the "second stage" moment condition, Assumptions (1) and (2) imply we can decompose $\omega_{i t}$ into its conditional expectation at time $t-1$, and an innovation term, i.e.

$$
\omega_{i t}=E\left[\omega_{i t} \mid I_{i t-1}\right]+\xi_{i t}=E\left[\omega_{i t} \mid \omega_{i t-1}\right]+\xi_{i t}=g\left(\omega_{i t-1}\right)+\xi_{i t}
$$

where by construction $E\left[\xi_{i t} \mid I_{i t-1}\right]=0$. Substituting this into the production function gives

$$
\begin{aligned}
y_{i t} & =\beta_{0}+\beta_{k} k_{i t}+\beta_{l} l_{i t}+g\left(\omega_{i t-1}\right)+\xi_{i t}+\epsilon_{i t} \\
& =\beta_{0}+\beta_{k} k_{i t}+\beta_{l} l_{i t}+g\left(\Phi_{t-1}\left(k_{i t-1}, i_{i t-1}\right)-\beta_{0}-\beta_{k} k_{i t-1}\right)+\xi_{i t}+\epsilon_{i t}
\end{aligned}
$$

where the second line follows from the definition of $\Phi_{t}\left(k_{i t}, i_{i t}\right)$.

\footnotetext{
${ }^{2}$ If firm-specific variables such as exogenous labor input prices are observed by the econometrician, they can be included in (2). However, the premise of most of this literature is that such variables are either not available or not believed to be exogenous. If one observed exogenous, across-firm, variation in all input prices, estimating the production function using input price based IV methods might be preferred to OP/LP related methodology (due to fewer auxiliary assumptions). In other words, OP/LP (and the methods we suggest) are probably most useful when one does not observe any exogenous across-firm variation in input prices, or when one observes exogenous across-firm variation in input prices for only a subset of the inputs (for the latter, see, e.g., Doraszelski and Jaumandreu $(2013))$.
} 
Given that $E\left[\xi_{i t} \mid I_{i t-1}\right]=0$ and $E\left[\epsilon_{i t} \mid I_{i t}\right]=0$, the second stage of OP's estimation procedure uses the following moment condition:

$$
E\left[\xi_{i t}+\epsilon_{i t} \mid I_{i t-1}\right]=E\left[y_{i t}-\beta_{0}-\beta_{k} k_{i t}-\beta_{l} l_{i t}-g\left(\Phi_{t-1}\left(k_{i t-1},, i_{i t-1}\right)-\beta_{0}-\beta_{k} k_{i t-1}\right) \mid I_{i t-1}\right]=0
$$

where estimation proceeds by "plugging in" the first stage estimates of $\widehat{\beta}_{l}$ and $\widehat{\Phi}_{t-1}$ into the second stage moment. Note that the two sets of moments, (5) and (8), that identify the production function parameters and the functions $\Phi_{t}$ and $g$ can be thought of in the semiparametric moment restriction framework of Ai and Chen (2003) (and as pointed out by Wooldridge (2009), could be thought of as IV estimators). While these moment conditions could be estimated jointly as in Ai and Chen or Wooldridge (2009), estimation using a two-stage "plug-in" approach is consistent (see Ai and Chen (2007)) and popular in the empirical literature for computational reasons. Joint estimation requires a numeric, non-linear search over all the parameters of the model (including those representing the functions $\Phi_{t}$ and $g$ ), which can be time-consuming and/or unreliable. The two-stage approach requires at most two sequential smaller dimensional searches, and if $\Phi_{t}$ is specified using polynomials or kernels, the first stage estimation of $\beta_{l}$ and $\Phi_{t-1}$ can be done analytically. On the other hand, as pointed out by Wooldridge (2009), there are advantages to estimating the moment conditions jointly, e.g. potential efficiency gains from information in the covariances of the moments (though this is not necessarily the case, see Ackerberg, Chen, Hahn and Liao (2014)), and more straightforward asymptotic standard errors (see Chen, Hahn, Liao, and Ridder (2013)).

LP take a related approach. The key difference is that instead of using the investment demand equation to "invert" out $\omega_{i t}$, they use the demand function for an intermediate input $m_{i t}$ (e.g. electricity, fuel, or materials). More specifically, they consider the following production function

$$
y_{i t}=\beta_{0}+\beta_{k} k_{i t}+\beta_{l} l_{i t}+\beta_{m} m_{i t}+\omega_{i t}+\epsilon_{i t}
$$

and replace Assumptions (4) and (5) with

Assumption 6 Assumption 4b: (Scalar Unobservable) Firms' intermediate input demand is given by

$$
m_{i t}=f_{t}\left(k_{i t}, \omega_{i t}\right)
$$

Assumption 7 Assumption 5b: (Strict Monotonicity) $f_{t}\left(k_{i t}, \omega_{i t}\right)$ is strictly increasing in $\omega_{i t}$.

Assumptions (4b) and (5b) again allow one to invert $\omega_{i t}$ as a function of observables, i.e. $\omega_{i t}=f_{t}^{-1}\left(k_{i t}, m_{i t}\right)$, analagously to the above. Assumption (4b) is consistent with a model in which $l_{i t}$ and $m_{i t}$ are non-dynamic inputs that are chosen simultaneously at $t$, after the firm has observed $\omega_{i t}$ - what is crucial is that $\omega_{i t}$ is the only unobservable entering the intermediate input demand function. Like in OP, this places restrictions on the underlying model, e.g. that firms operate in the same labor and material input markets (or identical labor and material input markets), and that they operate in the same output market with either homogeneous goods or completely symmetric product differentiation (or identical output markets). Given these restrictions, Assumption (5b) is verified by LP under mild assumptions on primitives.

Other than this, LP proceeds identically to OP. The first stage involves using the equation

$$
\begin{aligned}
y_{i t} & =\beta_{0}+\beta_{k} k_{i t}+\beta_{l} l_{i t}+\beta_{m} m_{i t}+f_{t}^{-1}\left(k_{i t}, m_{i t}\right)+\epsilon_{i t} \\
& =\beta_{l} l_{i t}+\Phi_{t}\left(k_{i t}, m_{i t}\right)+\epsilon_{i t}
\end{aligned}
$$


and the moment condition

$$
E\left[\epsilon_{i t} \mid I_{i t}\right]=E\left[y_{i t}-\beta_{l} l_{i t}-\Phi_{t}\left(k_{i t}, m_{i t}\right) \mid I_{i t}\right]=0
$$

to obtain estimates $\widehat{\beta}_{l}$ and $\widehat{\Phi}_{t}\left(k_{i t}, m_{i t}\right)$. These estimates are plugged in to the second stage moment condition

$$
E\left[\xi_{i t}+\epsilon_{i t} \mid I_{i t-1}\right]=E\left[y_{i t}-\beta_{0}-\beta_{k} k_{i t}-\beta_{l} l_{i t}-\beta_{m} m_{i t}-g\left(\Phi_{t-1}\left(k_{i t-1}, m_{i t-1}\right)-\beta_{0}-\beta_{k} k_{i t-1}-\beta_{m} m_{i t-1}\right) \mid I_{i t-1}\right]=0
$$

to obtain estimates $\widehat{\beta}_{0}, \widehat{\beta}_{k}$, and $\widehat{\beta}_{m}$. LP suggest bootstrapping to obtain standard errors, while Pakes and Olley (1995) provide analytic standard error estimators.

\subsection{Discussion}

As noted by Levinsohn and Petrin, there are a number of advantages to using an intermediate input $m_{i t}$ rather than investment $i_{i t}$ as a way of "inferring" unobserved productivity. First, theoretically it can be considerably easier to verify Assumption (5b) than Assumption (5) - verifying (5) requires analysis of a dynamic programming problem, but as intermediate inputs are typically assumed to be non-dynamic inputs, verifying (5b) does not. Second, in practice investment data can be lumpy, e.g. in firm or plant level datasets one often sees $i_{i t}=0$. This casts doubt on Assumption (5) (strict monotonicity), at least for the observations where $i_{i t}=0$. While the OP procedure can be adapted to work in this weakly monotonic situation, it requires discarding data with zero investment, which LP note can sometimes be a significant portion of the data. Moreover, lumpiness might also occur at points other than $i_{i t}=0$, which is more problematic.

Another important advantage of LP using an intermediate input is related to the critique by Griliches and Mairesse (1998). In OP, Assumption (4) rules out any firm-specific unobservables (other than the productivity shock $\omega_{i t}$ ) affecting investment demand. This, for example, rules out unobserved capital adjustment costs that vary across firms, as well as unobserved, firm-specific shocks to investment prices. The LP method does not rule such shocks out - since the intermediate input and labor are non-dynamic inputs that only affect current profits, there is no reason for $m_{i t}$ to depend on these shocks (conditional on $k_{i t}$ and $\omega_{i t}$ ). Of course, LP has an analagous restriction regarding unobserved, firm-specific shocks to the price of intermediate inputs and labor. However, this distinction is not symmetric, since OP also rules out these material or labor price shocks (except for the special case where they are independent across time). The reason is that if, e.g., unobserved wage shocks are serially correlated, the current wage is related to the marginal revenue product of capital in the future, and thus current investment should depend on the current wage, violating Assumption (5). So in summary, neither OP nor LP allow serially correlated, unobserved heterogeneity (across firms) in prices of labor or intermediate inputs, while only OP rules out unobserved heterogeneity (across firms) in the price of investment or capital adjustment costs.

Lastly, note that one can extend these methods to situations where the econometrician actually observes firmspecific variation in the prices of inputs. If these are assumed exogenous, OP/LP can be applied - the prices can simply be included in the appropriate input demand functions above and used as instruments. For example, if exogenous variation in $p_{i t}^{m}$ and $p_{i t}^{l}$ across firms is observed, these variables should be included in the intermediate input equation, i.e. $m_{i t}=f_{t}\left(k_{i t}, \omega_{i t}, p_{i t}^{m}, p_{i t}^{l}\right)$, and included in the instrument set $I_{i t}$. That said, both OP/LP make many auxiliary assumptions, so if one observed exogenous, firm-specific variation in all input prices, it might be preferrable to use simple "input price based IV" methods, i.e. regress $y_{i t}$ on $k_{i t}, l_{i t}$, and $m_{i t}$ using $p_{i t}^{k}, p_{i t}^{l}$ and $p_{i t}^{m}$ as instruments. This would provide consistent estimates without the OP/LP auxiliary assumptions. Of course, the observed price differences across firms need to be exogenous, i.e. the differences cannot capture input quality differences or different firms' choices of location on a downward sloping input supply curve. Having credibly exogenous, observed, variation in the costs of all inputs is fairly rare. Perhaps more likely is a case where one observes exogenous variation in prices of a subset of the instruments. In this case, pure "input price based IV" 
methods are generally not possible, and we feel that techniques related to OP/LP are valuable. Doraszelski and Jaumandreu (2013) estimate a model where this is the case. ${ }^{3}$

\section{Functional Dependence Problems}

This paper argues that even if the above assumptions hold, there are identification problems with these methodologies. The issue is one of functional dependence in the first step of the respective procedures. We illustrate these issues in the LP context, though one can make very similar arguments in the OP context. To give a brief intuition behind the result, consider a parametric version of the first stage of LP, i.e. where instead of treating (10) (and its inverse) non-parametrically, one actually uses the parametric FOC corresponding to a Cobb-Douglas production function. The first order condition for $m_{i t}$ (conditional on $k_{i t}, l_{i t}$, and $\omega_{i t}$ ) is

$$
\beta_{m} K_{i t}^{\beta_{k}} L_{i t}^{\beta_{l}} M_{i t}^{\beta_{m}-1} e^{\omega_{i t}}=\frac{p_{m}}{p_{y}}
$$

assuming firms are price takers in both input and output markets. ${ }^{4}$ Inverting this equation for $\omega_{i t}$ and substituting into the production function results in:

$$
y_{i t}=\ln \left(\frac{1}{\beta_{m}}\right)+\ln \left(\frac{p_{m}}{p_{y}}\right)+m_{i t}+\epsilon_{i t}
$$

Since $\beta_{l}$ does not enter this expression, a moment condition in $\epsilon_{i t}$ based on this equation (which is analagous to the first stage of LP) cannot be informative on $\beta_{l}{ }^{5}$

While the above result might not hold for more general functional forms of the production function, the LP and OP procedures treat the inverted FOCs non-parametrically, which makes identification tougher. The first step moment condition (13) used to estimate $\beta_{l}$ in LP is equivalent to the partially linear model studied by Robinson (1988). His condition for identification of $\beta_{l}$ (Equation 3.5, see also Ai and Chen (2003) Assumption 3.3 and discussion) is that

$$
E\left[\left\{l_{i t}-E\left[l_{i t} \mid k_{i t}, m_{i t}, t\right]\right\}\left\{l_{i t}-E\left[l_{i t} \mid k_{i t}, m_{i t}, t\right]\right\}^{\prime}\right] \text { is positive definite }
$$

An immediate observation is that if $l_{i t}$ is functionally dependent on $k_{i t}, m_{i t}$, and $t$ (i.e. $l_{i t}$ is only a function of $k_{i t}, m_{i t}$, and $t$ ), this condition does not hold. Intuitively, the problem here is that. $l_{i t}$ is fully determined by the values of $k_{i t}, m_{i t}$, and $t$, so there is no source of variation in $l_{i t}$ (conditional on $k_{i t}, m_{i t}$, and $t$ ) to identify $\beta_{l}$. So we focus our discussion on investigating possible data-generating-processes for $l_{i t}$ and ask the question whether these DGPs generate this functional dependence.

Perhaps the simplest possible DGP for $l_{i t}$ is analogous to that for $m_{i t}$ (equation (10)), i.e.

$$
l_{i t}=h_{t}\left(k_{i t}, \omega_{i t}\right)
$$

This assumes that like $m_{i t}$, labor has no dynamic implications and is chosen with full knowledge of $\omega_{i t}$. With this

\footnotetext{
${ }^{3}$ In contrast to OP and LP, they treat the inverted input demand function parametrically. They have two good reasons for this. First, including observed input prices in the inverted function increase the dimensionality of the function and make non-parametric treatment more challenging. Second, since the production function has already been modelled parametrically, one can obtain parametric forms of input demand fuctions for non-dynamic inputs without significant auxiliary assumptions. Doraszelski and Jaumandreu also extend the OP/LP model to one in which observed firm R\&D expenditures affect the evolution of $\omega_{i t}$, an important idea that could also be applied in the context of our estimation procedure.

${ }^{4}$ This version of the first order condition assumes that $\epsilon_{i t}$ is pure measurement error in $y_{i t}$ (if $\epsilon_{i t}$ was an unanticipated shock to output, the expectation of $\exp \left(\epsilon_{i t}\right)$ would enter the first order condition as well).

${ }^{5}$ The above analysis uses the choice of $m_{i t}$ conditional on levels of $k_{i t}, l_{i t}$, and $\omega_{i t}$. This is most naturally interpreted in the case where $m_{i t}$ is chosen after $l_{i t}$. One obtains the same result if one solves simultaneously for optimal choices of both $m_{i t}$ and $l_{i t}$ conditional on levels of $k_{i t}$ and $\omega_{i t}$, and plugs the inverted FOC for $m_{i t}$ into the production function.
} 
DGP, substituting the inverted (10) into (17) results in

$$
l_{i t}=g_{t}\left(k_{i t}, f_{t}^{-1}\left(k_{i t}, m_{i t}\right)\right)
$$

which states that $l_{i t}$ is functionally dependent on $k_{i t}, m_{i t}$, and $t$, and implies that the identification condition (16) fails. Intuitively, in equation (12), the contribution of labor to output cannot be separately identified from the non-parametric function $\Phi_{t}\left(k_{i t}, m_{i t}\right)$.

That said, while (17) might be the simplest specification for the data generation process (DGP) for $l_{i t}$, it is not the only possibility. Our goal now is to search for an alternative DGP for $l_{i t}$ (and possibly for $m_{i t}$ ) that does not suffer this functional dependence problem and is consistent with the basic assumptions of the LP (and OP) procedure(s).

First, consider adding firm-specific input prices to the above model of input choice, e.g. prices of labor $\left(p_{i t}^{l}\right)$ and materials $\left(p_{i t}^{m}\right)$. Generally speaking, both of these firm-specific input prices will affect a firm's choices of both $l_{i t}$ and $m_{i t}$ (i.e. conditional on $p_{i t}^{m}$, decreasing $p_{i t}^{l}$ will generally increase a firm's optimal level of both $l_{i t}$ and $m_{i t}$.) Therefore, these input prices would need to be observed by the econometrician, because otherwise (10) would violate the scalar unobservable assumption necessary for the LP inversion. Since intermediate input demand depends on these firm-specific input prices, they end up in the first step non-parametric function, i.e.

$$
E\left[\epsilon_{i t} \mid I_{i t}\right]=E\left[y_{i t}-\beta_{l} l_{i t}-\Phi_{t}\left(k_{i t}, m_{i t}, p_{i t}^{l}, p_{i t}^{m}\right) \mid I_{i t}\right]=0
$$

and there is again a functional dependence problem, as there are no variables that shift $l_{i t}$ but do not enter $\Phi_{t}$. Another possibility, allowing labor to have dynamic effects, doesn't help for the same reason. $l_{i t-1}$ becomes a state variable of the problem, but since both $m_{i t}$ and $l_{i t}$ both depend on $l_{i t-1}$, there is still functional dependence.

One way to eliminate the functional dependence problem is to assume that there is "optimization error" in $l_{i t}$. By optimization error, we mean that there is a optimal level of $l_{i t}$ (e.g. given by (17)), but that for some reason the firm chooses that optimal level plus noise (that is independent over time and of other errors in the model). One example of this could be workers calling in sick. ${ }^{6}$ Such optimization error induces variation in $l_{i t}$ conditional on $k_{i t}, m_{i t}$, and $t$, and hence the first step can consistently estimate $\beta_{l}$. However, note that the methodology is not compatible with similar optimization error in $m_{i t}$, since this would violate the scalar unobservable assumption required to invert (10) and obtain $\omega_{i t}$. The context of sick days is a good way to illustrate this point with some applied relevance. Suppose that $m_{i t}$ are material inputs used in production and that when workers are out sick, less of these materials are used. If the econometrician's measure of $m_{i t}$ is the actual level of material input used (which is negatively affected by sick days), there is implicitly optimization error in both $l_{i t}$ and $m_{i t}$, and because of this optimization error in $m_{i t}$ the first step will not produce consistent estimates of $\beta_{l}$. However, if the econometrician's measure of $m_{i t}$ is the planned level of material input used (i.e. planned/ordered prior to the realization of sick days, and thus unaffected by sick days), there is only optimization error in $l_{i t}$ and the first step will produce consistent estimates of $\beta_{l}$. In contrast, the i.i.d. "optimization error" in $l_{i t}$ DGP might not be a good representation of a situation where firms' labor choices are distorted by exogenous union issues. This is because union issues are unlikely to be i.i.d. over time, and hence either planned or used material inputs are likely to respond to the (unobserved) union shocks, violating the scalar unobservable assumption. Note that classical measurement error in $l_{i t}$ (i.e. noise in observed labor that does not affect output) is problematic because of standard measurement error problems, and classical measurement error in $m_{i t}$ is problematic because it again violates the scalar unobservable assumption that is crucial for the inversion.

A second, similar, DGP that can eliminate the functional dependence problem involves changing the assumption

\footnotetext{
${ }^{6}$ Note the difference between our "optimization error" and the "optimization error" described by Mundlak (1996). We are simply adding exogenous noise to the desired input level (workers exogenously calling in sick). Mundlak describes "optimization error" due to incomplete information, for example, firms choose labor input based on an expected price of labor, but the realized price of labor is different, making their choice of labor input seem suboptimal ex post.
} 
that $l_{i t}$ and $m_{i t}$ are chosen with the same information set. Consider a point in time, $t-b$, sometime between period $t-1$ and $t$ (i.e. $0<b<1$ ). ${ }^{7}$ First, note that if one assumes $l_{i t}$ is chosen at $t-b$ and $m_{i t}$ is chosen at $t$, there is a problem because in such a model, optimal $m_{i t}$ will generally directly depend on the previously chosen level of $l_{i t}$. Since $l_{i t}$ enters (10), the first step moment becomes

$$
E\left[\epsilon_{i t} \mid I_{i t}\right]=E\left[y_{i t}-\beta_{l} l_{i t}-\Phi_{t}\left(k_{i t}, l_{i t}, m_{i t}\right) \mid I_{i t}\right]=0
$$

which obviously precludes identification of $\beta_{l}$ in the first step. However, one can alternatively consider the reverse situation, i.e. where $m_{i t}$ is chosen at $t-b$ and $l_{i t}$ is chosen at $t$. Suppose there is some unanticipated firm-specific shock to the price of labor (or demand), $\varkappa_{i t}$, that is realized between the points $t-b$ and $t$ (i.e. it is not in the firm's information set $\left(I_{i t-b}\right)$ when $m_{i t}$ is chosen, but it is in the firm's information set $\left(I_{i t}\right)$ when $l_{i t}$ is chosen). Such a shock will vary $l_{i t}$ independently of $k_{i t}, m_{i t}$, and $t$, and thus will eliminate the functional dependence problem. This shock $\varkappa_{i t}$ does not need to be observed by the econometrician, but if it is unobserved, it must be i.i.d. over time (otherwise the optimal $m_{i t+1}$ will depend on $\varkappa_{i t}$, violating the scalar unobservable assumption). Another assumption that is required in this DGP is that the firm observes $\omega_{i t}$ when choosing $m_{i t}$ at time $t-b$ (perhaps more intuitively that $\omega$ does not evolve between time $t-b$ and time $t$ ). Otherwise the non-parametric function of $m_{i t}$ and $k_{i t}$ will not perfectly control for $\omega_{i t}$ in the moment condition.

These functional dependence problems (and possible assumptions that avoid them) generalize to the first step of the OP procedure that alternatively uses the investment function to control for productivity variation.. Firm specific input prices (observed or unobserved) and dynamics in $l_{i t}$ generally do not break the functional dependence, but optimization error in $l_{i t}$ or i.i.d, firm-specific wage (or output price) shocks after $i_{i t}$ is chosen do. There is one additional DGP that breaks the functional dependence problem in the OP setup. Continuing with the above setup with the "subperiod" $t-b$, assume that $\omega$ evolves between $t-1, t-b$, and $t$ according to a first order Markov process, i.e.

$$
p\left(\omega_{i t-b} \mid I_{i t-1}\right)=p\left(\omega_{i t-b} \mid \omega_{i t-1}\right)
$$

and

$$
p\left(\omega_{i t} \mid I_{i t-b}\right)=p\left(\omega_{i t} \mid \omega_{i t-b}\right)
$$

Suppose also that $l_{i t}$ is chosen at time $t-b$ with information set $I_{i t-b}$, while $i_{i t}$ is chosen at time $t$ with information set $I_{i t}$

In this case, a firm's optimal investment will follow

$$
i_{i t}=f_{t}\left(k_{i t}, \omega_{i t}\right)
$$

while a firm's optimal labor input will not be a function of $\omega_{i t}$, but of $\omega_{i t-b}$, i.e.

$$
l_{i t}=g_{t}\left(\omega_{i t-b}, k_{i t}\right)
$$

Since $\omega_{i t-b}$ cannot generally be written as a function of $k_{i t}$ and $i_{i t}, l_{i t}$ will not generally be functionally dependent on $k_{i t}$ and $i_{i t}$, allowing consistent estimation of $\beta_{l}$ in the first step. Note the intution behind this - labor is chosen without perfect information about what $\omega_{i t}$ is, and this incomplete information is what generates variation in $l_{i t}$ conditional on the non-parametric function $f_{t}^{-1}\left(k_{i t}, i_{i t}\right)$. However, note that this DGP does need to rule out a firm's

\footnotetext{
${ }^{7}$ Note that we continue to assume that production occurs "on the period", i.e. at periods $t-1$ and $t$. Note that this intermediate period, $t-b$, does not need to have a literal interpretation in terms of time. What we are simply trying to do is allow different inputs to be chosen with different information sets, and with this heuristic device, an "input chosen at $t$ " is chosen with more information than is an "input chosen at $t-b$ ", which is chosen with more information than is an "input chosen at $t-1$ ".
} 
choice of $l_{i t}$ having dynamic implications. If labor did have dynamic effects, then $l_{i t}$ would directly impact a firm's choice of $i_{i t}$. As a result, $l_{i t}$ would directly enter the first stage non-parametric function and prevent identification of $\beta_{l}$ in that first stage. ${ }^{8}$

\subsection{Discussion}

To summarize, it appears that the first stage of the OP and LP procedures correctly identify $\beta_{l}$ only under three very specific DGPs - 1) i.i.d. optimization error in $l_{i t}$ (and not in $m_{i t}\left(\right.$ or $\left.i_{i t}\right)$ ) 2) i.i.d. shocks to the price of labor or output after $m_{i t}\left(\right.$ or $i_{i t}$ ) is chosen but prior to $l_{i t}$ being chosen, or 3 ) (in the OP context) labor is non-dynamic and chosen at $t-b$ as a function of $\omega_{i t-b}$, while $i_{i t}$ is chosen at $t$. Note that in practice, one probably would not literally observe the functional dependence problem described above (if one were using polynomials to approximate the non-parametric function, functional dependence would arise in the form of collinearity between $l_{i t}$ and the terms in the polynomial approximation.) In other words, it is likely that estimation of (5) or (13) will produce an actual numerical estimate of $\beta_{l}$. Our point is that unless one believes that one of these three DGPs is generating the data (and additionally that these DGPs are the only reasons why there is no functional dependence), this is simply not a consistent estimator of $\beta_{l}$.

\section{Our Alternative Procedure}

We now propose an alternative estimation procedure that avoids the functional dependence problems discussed above. Perhaps just as importantly, it also relaxes other assumptions typically made in applications of OP and LP. Specifically, our model allows there to be exogenous, serially correlated, unobserved firm-specific shocks to the price of labor, or firm-specific unobserved adjustment costs to the labor input. It also allows the labor input to have dynamic effects (e.g. hiring or firing costs) more generally. We emphasize that the approach is only a slight adaptation of the OP/LP methodologies, and essentially relies the same moment conditions. The main difference between our approach and OP and LP is that in our approach, we invert "conditional" rather than "unconditional" input demand functions to control for unobserved productivity. This results in a first stage that does not identify the coefficients on variable inputs (e.g. labor). Instead, all coefficients are all estimated in the second stage. However, as we shall see, the first stage will still be important to 'net out' the untransmitted error $\epsilon_{i t}$ from the production function. We start by showing how our method works using the LP intermediate input function before showing the extension to using the OP investment function.

\subsection{The Basic Procedure}

Consider the following 'value-added' production function

$$
y_{i t}=\beta_{0}+\beta_{k} k_{i t}+\beta_{l} l_{i t}+\omega_{i t}+\epsilon_{i t}
$$

with the alternative assumptions

\section{Assumption 8}

\footnotetext{
${ }^{8}$ Note why this DGP does not solve the functional dependence problem in the context of the LP model. In the LP model, if $l_{i t}$ is chosen before $m_{i t}$, then $m_{i t}$ will directly depend on $l_{i t}$, making $\beta_{l}$ unidentified in the first stage. In OP, even if $l_{i t}$ is chosen before $i_{i t}$, $i_{i t}$ does not depend on $l_{i t}$ (as long as one maintains the assumption that labor is non-dynamic). This is because $i_{i t}$, unlike $m_{i t}$, is not directly linked to period $t$ outcomes, and thus $l_{i t}$ will not affect a firm's optimal choice of $i_{i t}$.
} 
Assumption 9 Assumption 3c: (Timing of Input Choices) Firms accumulate capital according to

$$
k_{i t}=\kappa\left(k_{i t-1}, i_{i t-1}\right)
$$

where investment $i_{i t-1}$ is chosen in period $t-1$. Labor input $l_{i t}$ has potential dynamic implications and is chosen at period $t$, period $t-1$, or period $t-b$ (with $0<b<1)$

Assumption 10 Assumption 4c: (Scalar Unobservable) Firms' intermediate input demand is given by

$$
m_{i t}=\widetilde{f}_{t}\left(k_{i t}, l_{i t}, \omega_{i t}\right)
$$

Assumption 11 Assumption 5c: (Strict Monotonicity) $\tilde{f}_{t}\left(k_{i t}, l_{i t}, \omega_{i t}\right)$ is strictly increasing in $\omega_{i t}$.

First, note that we consider a 'value added' production function in the sense that the intermediate input $m_{i t}$ does not enter the production function to be estimated. One interpretation of this is that the gross output production function is Leontief in the intermediate input, where this intermediate input is proportional to output (e.g. see our Monte-Carlo specification). Another follows the existing literature on value-added production functions, see, e.g. Bruno (1978) and Diewert (1978). As discussed in those papers, as well as in Basu and Fernald (1997), the assumptions behind the existence of a meaningful value-added production function are subtle and non-trivial. We would not suggest applying our procedure to gross output production functions that are not Leontief in the intermediate input(s) (i.e. production functions where $m_{i t}$ enters (23)). This is because Bond and Söderbom (2005) (for the Cobb-Douglas case) and Gandhi, Navarro, and Rivers (2014) (for the general case) have shown that under the scalar unobservable assumptions of OP, LP, and our procedure, these gross output production functions are not identified without imposing further restrictions of the model. If one is interested in doing this, it is likely preferable to use an approach where OP/LP-like moments are augmented by first order conditions or revenue share equations, e.g. LP (Appendix B) and Doraszelski and Jaumandreu (2013), who do this in the context of a Cobb-Douglas production function, or Gandhi, Navarro, and Rivers (2014), who show how to do this much more generally.

Moving to the assumptions of the model, Assumption (3c) generalizes Assumption (3) by allowing labor to have dynamic implications, i.e. choice of $l_{i t}$ not only affects current profits, but future profits, e.g. through hiring or firing costs. Note that this implies that $l_{i t}$ is part of the state space of the firm's dynamic problem. We can allow $l_{i t}$ to be chosen at period $t$ (and thus a function of $I_{i t}$ ), chosen at period $t-1$ (and thus a function of only $I_{i t-1}$ ), or at some point in-between (with an intermediate information set)

Assumption (4c) represents the crucial conceptual difference between our procedure and LP. The difference between Assumption (4b) and Assumption (4c) is that we are using a conditional input demand function to control for unobserved productivity, while LP uses an unconditional (or less conditional) input demand function. More precisely, (24) is the input demand function for $m_{i t}$ conditional on $l_{i t}$, while (10) is the input demand function for $m_{i t}$ not conditional on $l_{i t}$. While our conditional input demand function might be most naturally thought of as representing a situation where $m_{i t}$ is chosen after $l_{i t}$, it can equally well represent a situation where $m_{i t}$ and $l_{i t}$ are chosen simultaneously. ${ }^{9}$ As does Assumption (4) and (4b), Assumption (4c) rules out production functions with multiple structural unobservables like McElroy (1987). Assumption (5c) is the strict monotonicity assumption

\footnotetext{
${ }^{9}$ There is no loss of generality in representing $\max _{l_{i t}, m_{i t}}\{\cdot\}$ as $\max _{l_{i t}}\left\{\max _{m_{i t} \mid l_{i t}}\{\cdot\}\right\}$. Note that LP's unconditional (on $l_{i t}$ ) intermediate input demand equation, i.e. $m_{i t}=f_{t}\left(k_{i t}, \omega_{i t}\right)$, is most naturally interpreted as a situation where $l_{i t}$ and $m_{i t}$ are chosen simultaneously as a function of the current state $\left(k_{i t}, \omega_{i t}\right)$.
} 
necessary for the inversion - since $m_{i t}$ is still a non-dynamic input, it is straightforward to apply the invertibility proof of LP to obtain this result.

Given these assumptions, we can follow LP, invert intermediate input demand $\omega_{i t}=\tilde{f}_{t}^{-1}\left(k_{i t}, l_{i t}, m_{i t}\right)$ and substitute into the production function, i.e.

$$
y_{i t}=\beta_{0}+\beta_{k} k_{i t}+\beta_{l} l_{i t}+\tilde{f}_{t}^{-1}\left(k_{i t}, l_{i t}, m_{i t}\right)+\epsilon_{i t}=\widetilde{\Phi}_{t}\left(k_{i t}, l_{i t}, m_{i t}\right)+\epsilon_{i t}
$$

Since we follow LP and treat $\tilde{f}_{t}^{-1}$ non-parametrically, the first three terms are clearly not identified and are subsumed into $\widetilde{\Phi}_{t}\left(k_{i t}, l_{i t}, m_{i t}\right)=\beta_{0}+\beta_{k} k_{i t}+\beta_{l} l_{i t}+\omega_{i t}$, resulting in the following first stage moment condition

$$
E\left[\epsilon_{i t} \mid I_{i t}\right]=E\left[y_{i t}-\widetilde{\Phi}_{t}\left(k_{i t}, l_{i t}, m_{i t}\right) \mid I_{i t}\right]=0
$$

Unlike LP, (26) does not permit estimation of $\beta_{l}$ in the first stage. However, it does produce an estimate $\widehat{\widetilde{\Phi}}_{t}\left(k_{i t}, l_{i t}, m_{i t}\right)$ of $\widetilde{\Phi}_{t}\left(k_{i t}, l_{i t}, m_{i t}\right)$. We propose estimating $\beta_{l}$ along with the other production function parameters in the second stage using the following second stage conditional moment.

$$
E\left[\xi_{i t}+\epsilon_{i t} \mid I_{i t-1}\right]=E\left[y_{i t}-\beta_{0}-\beta_{k} k_{i t}-\beta_{l} l_{i t}-g\left(\widetilde{\Phi}_{t-1}\left(k_{i t-1}, l_{i t-1}, m_{i t-1}\right)-\beta_{0}-\beta_{k} k_{i t-1}-\beta_{l} l_{i t-1}\right) \mid I_{i t-1}\right]=0
$$

where $\widetilde{\Phi}_{t-1}$ is replaced by its estimate from the first stage. Note that this moment condition is essentially identical to that used by LP (i.e. equation (14)), except for the fact that since $\widetilde{\Phi}_{t-1}$ now contains the labor term, it also needs to be subtracted out inside the $g$ function.

As usual, it is easiest to transform conditional moments into unconditional moments for actual estimation. It is important to note that since our second stage requires estimating an additional parameter $\left(\beta_{l}\right)$ as compared to LP, we require an additional unconditional moment relative to LP. To illustrate, consider a simple model where $\omega_{i t}=\rho \omega_{i t-1}+\xi_{i t}$. Suppose the first stage is performed by OLS regression of $y_{i t}$ on a high-order polynomial in $\left(k_{i t}, l_{i t}, m_{i t}\right)$ to obtain $\widehat{\widetilde{\Phi}}_{t}\left(k_{i t}, l_{i t}, m_{i t}\right)$. If labor is assumed to be chosen after time $t-1$ (implying $l_{i t}$ will generally be correlated with $\xi_{i t}$ ), a natural set of four second stage moment conditions to estimate the three production function parameters $\left(\beta_{0}, \beta_{k}\right.$, and $\left.\beta_{l}\right)$ and $\rho$ is

$$
E\left[\left(y_{i t}-\beta_{0}-\beta_{k} k_{i t}-\beta_{l} l_{i t}-\rho \cdot\left(\widetilde{\Phi}_{t-1}\left(k_{i t-1}, l_{i t-1}, m_{i t-1}\right)-\beta_{0}-\beta_{k} k_{i t-1}-\beta_{l} l_{i t-1}\right)\right) \otimes\left(\begin{array}{cc}
1 \\
k_{i t} \\
l_{i t-1} \\
\widetilde{\Phi}_{t-1}\left(k_{i t-1}, l_{i t-1}, m_{i t-1}\right)
\end{array}\right)\right]=0
$$

In contrast, second stage LP estimation based on (14) only requires three moments, though as exemplified in the LP empirical work, one could alternatively utilize all four moments (or more than four using other components of $\left.I_{i t-1}\right)$ and have overidentifying restrictions. ${ }^{10}$

In some industries, one might be willing to assume that labor is chosen by the firm at $t-1$, i.e. $l_{i t} \in I_{i t-1}$ (or alternatively make the assumption that $\omega_{i t}$ is not observed by the firm until period $\left.t+1\right)$. This is a potentially strong assumption, but it might be plausible in situations where there are significant hiring or firing costs, or labor market rigidities, possibly due to government regulation. In this case, one could add $l_{i t}$ to the information set (or

\footnotetext{
${ }^{10}$ There are many ways to estimate the model based on the second stage moments. For example, the Appendix in LP illustrates how one can do second step estimation with a non-linear search over just $\left(\beta_{k}, \beta_{l}\right)$. A similar approach can work here, where one only has to search over $\left(\beta_{k}, \beta_{l}\right)$ rather than over $\left(\beta_{0}, \beta_{k}, \beta_{l}\right)$ and the parameters of $g$ (see our Monte-Carlo code). Relatedly, a prior version of this paper illustrated the approach with a second step moment in $\xi_{i t}$ rather in $\xi_{i t}+\epsilon_{i t}$. As noted by Gandhi, Navarro, and Rivers (2014), the moment in $\xi_{i t}+\epsilon_{i t}$ can be used more generally, e.g. in some cases where the investment or intermediate input variable is only weakly monotonic in $\omega_{i t}$. Note that there are additional conditional moment conditions implied by the model related to inputs further in the past. Whether these are used as additional moments is typically a matter of preference.
} 
replace $l_{i t-1}$ with $l_{i t}$ in the instrument set if one wanted to keep the model exactly identified), i.e.

$$
E\left[\left(y_{i t}-\beta_{0}-\beta_{k} k_{i t}-\beta_{l} l_{i t}-\rho \cdot\left(\widetilde{\Phi}_{t-1}\left(k_{i t-1}, l_{i t-1}, m_{i t-1}\right)-\beta_{0}-\beta_{k} k_{i t-1}-\beta_{l} l_{i t-1}\right)\right) \otimes\left(\begin{array}{rr}
1 \\
k_{i t} \\
l_{i t} \\
l_{i t-1} \\
\widetilde{\Phi}_{t-1}\left(k_{i t-1}, l_{i t-1}, m_{i t-1}\right)
\end{array}\right)\right]=0
$$

This stronger assumption will generally lead to more precise estimates.

\subsection{Discussion}

In the context of the intermediate input function approach, we see a number of advantages of using our conditional intermediate input demands over LP's unconditional intermediate input demands. First, because we do not attempt to estimate $\beta_{l}$ in the first step, we avoid the functional dependence issues in the LP first stage. As a result, consistent estimates of $\beta_{l}$ do not rely on DGPs that involve optimization error in $l_{i t}$ (and none in $m_{i t}$ ), or i.i.d. firm-specific wage or output price shocks that are realized after the firm's choice of $m_{i t}$ (though our approach can provide consistent (but not as efficient) estimates of $\beta_{l}$ under such DGPs). Second, as noted above, this model is consistent with labor being a dynamic input. With such dynamics, in the case where $m_{i t}$ and $l_{i t}$ are chosen simultaneously, Assumption (4b) does not generally hold, since unconditional on $l_{i t}, m_{i t}$ will depend on $l_{i t-1}$. However, Assumption (4c) does hold, since conditional on $l_{i t}, l_{i t-1}$ should not affect a firm's optimal choice of $m_{i t}$.

Third, estimation using our moments (26) and (27) produces consistent estimates under some DGPs where the canonical LP moments (13) and (14) do not. One important example is a situation in which there is acrossfirm variation in exogenous wage conditions (or adjustment costs to dynamic labor) that is potentially serially correlated over time. Suppose that this wage (or adjustment cost) variation is not observed by the econometrician. In this case, the conditional intermediate input demand function does not depend on the wage/adjustment cost, i.e. conditional on $l_{i t}$, a firm's choice of $m_{i t}$ does not depend on the wage/adjustment cost. On the other hand firms' optimal choices of $m_{i t}$ given only $k_{i t}$, i.e. the unconditional intermediate input demand function, do generally depend on the unobserved wage/adjustment cost. In other words, Assumption (4c) does hold, while Assumption (4b) does not. Of course, it should be noted that if there are unobserved shocks to either prices of intermediate inputs or demand conditions, neither Assumption (4b) or Assumption (4c) hold, i.e. neither the conditional or unconditional approach will produce consistent estimates. To summarize with respect to the above and the discussion in Section 2.1, consider serially correlated, exogenous, unobserved shocks to the costs of $k_{i t}, l_{i t}$, and $m_{i t}$. OP cannot allow any of these shocks, LP can allow those relating to $k_{i t}$, but not those relating to $l_{i t}$ and $m_{i t}$, and our proposed moments can allow those relating to $k_{i t}$ and $l_{i t}$, but not those relating to $m_{i t}$.

\subsection{Extensions}

\subsubsection{Investment Function Approach}

One can also use our methodology with the investment demand function of OP, replacing the unconditional investment demand function $i=f_{t}\left(k_{i t}, \omega_{i t}\right)$ with a conditional (on $l_{i t}$ ) investment demand function $i_{i t}=f_{t}\left(k_{i t}, l_{i t}, \omega_{i t}\right.$ ). As above, this precludes identification of $\beta_{l}$ in the first stage - again, in our view this is a benefit as it avoids the first stage functional dependence issues. After a first stage that estimates $y_{i t}=\widetilde{\Phi}_{t}\left(k_{i t}, l_{i t}, i_{i t}\right)+\epsilon_{i t}$, the second stage moments

$$
E\left[\xi_{i t}+\epsilon_{i t} \mid I_{i t-1}\right]=E\left[y_{i t}-\beta_{0}-\beta_{k} k_{i t}-\beta_{l} l_{i t}-g\left(\widetilde{\Phi}_{t-1}\left(k_{i t-1}, l_{i t-1}, i_{i t-1}\right)-\beta_{0}-\beta_{k} k_{i t-1}-\beta_{l} l_{i t-1}\right) \mid I_{i t-1}\right]
$$


can be used to estimate the parameters. As when using the intermediate input demand function, this procedure is consistent with labor having dynamic effects. However, unlike when using the intermediate input demand function, it is not generally consistent with other unobservables entering either the $i_{i t}$ or $l_{i t}$ decisions. Any unobservable other than $\omega_{i t}$ that directly impacts investment, e.g. a firm specific shock to the price or adjustment costs of capital, is clearly problematic. Less obviously, serially correlated unobservables that affect the $l_{i t}$ decision, e.g. firm specific wage shocks, will generally also affect the $i_{i t}$ decision directly since $i_{i t}$ is a dynamic decision variable. For example, a firm facing low wages today expects to face low wages in the future, and hence invests more today. As a result, the inversion is problematic. The reason the intermediate input demand function approach of LP is more robust to these additional serially correlated unobservables is because intermediate inputs have no dynamic aspects to them, i.e. they only impact current output.

\subsubsection{Joint Estimation}

Wooldridge (2009) suggests an alternative implementation of the OP/LP moments that involves minimizing the first and second stage moments simultaneously. For example, in the LP model, he suggests estimating all the parameters simultaneously using the moment conditions

$$
E\left[\begin{array}{c}
\epsilon_{i t} \mid I_{i t} \\
\xi_{i t}+\epsilon_{i t} \mid I_{i t-1}
\end{array}\right]=E\left[\begin{array}{c}
y_{i t}-\beta_{l} l_{i t}-\Phi_{t}\left(k_{i t}, m_{i t}\right) \mid I_{i t} \\
y_{i t}-\beta_{0}-\beta_{k} k_{i t}-\beta_{l} l_{i t}-g\left(\Phi_{t-1}\left(k_{i t-1}, m_{i t-1}\right)-\beta_{0}-\beta_{k} k_{i t-1}\right) \mid I_{i t-1}
\end{array}\right]=0
$$

As pointed out by Wooldridge, there are several advantages to this approach. First, the joint approach avoids the functional dependence issue described above. Even if $l_{i t}$ is functionally dependent on $m_{i t}, k_{i t}$, and $t, \beta_{l}$ can be identified by the second set of moments. This highlights the fact that the functional dependence issue is not a problem with the moments used by OP and LP - it is a problem with how OP and LP use those moments sequentially, i.e. where one attempts to identify $\beta_{l}$ using only the first stage moments. Other advantages of the joint approach are potential efficiency gains, and simpler standard error calculations. There are also disadvantages of the joint approach - in particular, the joint approach requires a non-linear search over $\beta_{0}, \beta_{k}, \beta_{l}$, and the parameters representing the two unknown functions $\Phi_{t}$ and $g$. This is more time consuming and likely more error prone than the two-stage approach, which can often be performed with a non-linear search over just $\beta_{k}$ and $\beta_{l}$ (if $\Phi_{t}$ and $g$ are represented by polynomials, the first stage can be estimated using OLS, and in the second stage, OLS can be used to concentrate out the parameters of $g$ - see the Appendix).

Note that if one applied the idea of Wooldridge directly to our proposed moments (26) and (27), one gets something slightly different than (30), i.e.

$E\left[\begin{array}{c}\epsilon_{i t} \mid I_{i t} \\ \xi_{i t}+\epsilon_{i t} \mid I_{i t-1}\end{array}\right]=E\left[\begin{array}{c}y_{i t}-\widetilde{\Phi}_{t}\left(k_{i t}, l_{i t}, m_{i t}\right) \mid I_{i t} \\ y_{i t}-\beta_{0}-\beta_{k} k_{i t}-\beta_{l} l_{i t}-g\left(\widetilde{\Phi}_{t-1}\left(k_{i t-1}, l_{i t-1}, m_{i t-1}\right)-\beta_{0}-\beta_{k} k_{i t-1}-\beta_{l} l_{i t-1}\right) \mid I_{i t-1}\end{array}\right]=0$

Specifically, (30) is a restricted version of (31), a version where $\widetilde{\Phi}_{t}\left(k_{i t}, l_{i t}, m_{i t}\right)$ is assumed to be linear in $l_{i t}$. The distinction also exactly corresponds to our distinction between moments based on the unconditional intermediate input demand function (the Wooldridge moments (30)) and moments based on the conditional intermediate input demand function (31) . For the same reasons as discussed above, we prefer (31) because it makes fewer assumptions - (31) allows serially correlated unobserved wage shocks, allows dynamic labor with adjustment costs, and allows materials to be chosen after labor (e.g. labor chosen at $t-b$ as a function of $\omega_{i t-b}$ ). On the other hand, (30) does not allow this. One could also use the two sets of moments to test this restriction - if one models and interprets $\widetilde{\Phi}_{t}$ as a flexible parametric polynomial (rather than a non-parametric function), one could use a simple Wald or LR test for the restrictions that the linear coefficient on $l$ in $\widetilde{\Phi}(k, l, m)$ equals $\beta_{l}$ and all other coefficients involving $l$ equal 0 (note that this is related to the overidentifying restrictions tests performed by OP and LP). 


\subsubsection{Relation to Dynamic Panel Methods}

Interestingly, the form of our suggested estimators make them fairly easy to compare to estimators used in an alternative literature, the dynamic panel literature. This is important because up to now, researchers interested in estimating production functions have essentially been choosing between the OP/LP general approach versus the dynamic panel approach without a clear description of the similarities and differences of the identifying assumptions used in the two methods. We briefly discuss the differences and distinct advantages and disadvantages of both approaches.

As developed by work such as Chamberlain (1982), Anderson and Hsiao (1982), Arellano and Bond (1991), Arellano and Bover (1995), and Blundell and Bond (1998, 2000), the dynamic panel (DP) literature essentially extends the fixed effects literature to allow for more sophisticated error structures. We consider a simplified version of these models that is closest to our model.

$$
y_{i t}=\beta_{0}+\beta_{k} k_{i t}+\beta_{l} l_{i t}+\omega_{i t}+\epsilon_{i t}
$$

where $\omega_{i t}$ follows an $\operatorname{AR}(1)$ process, i.e. $\omega_{i t}=\rho \omega_{i t-1}+\xi_{i t}$. Like in our model, assume that $\epsilon_{i t}$ is i.i.d. over time and uncorrelated with $I_{i t}$ and that $\omega_{i t}$ is correlated with $k_{i t}$ and $l_{i t} \forall t$, but that the innovation $\xi_{i t}$, is uncorrelated with $I_{i t-1}$, i.e.all input choices prior to $t$.

The dynamic panel literature might proceed by " $\rho$-differencing" this model, i.e.

$$
y_{i t}-\rho y_{i t-1}=\beta_{0}(1-\rho)+\beta_{k}\left(k_{i t}-\rho k_{i t-1}\right)+\beta_{l}\left(l_{i t}-\rho l_{i t-1}\right)+\xi_{i t}+\left(\epsilon_{i t}-\rho \epsilon_{i t-1}\right)
$$

and estimating the model using the moment condition

$$
E\left[\xi_{i t}+\left(\epsilon_{i t}-\rho \epsilon_{i t-1}\right) \mid I_{i t-1}\right]=E\left[\left(y_{i t}-\rho y_{i t-1}\right)-\beta_{0}(1-\rho)-\beta_{k}\left(k_{i t}-\rho k_{i t-1}\right)-\beta_{l}\left(l_{i t}-\rho l_{i t-1}\right) \mid I_{i t-1}\right]=0
$$

This moment condition is similar to our proposed second stage moment condition $E\left[\xi_{i t}+\epsilon_{i t} \mid I_{i t-1}\right]=0$. What is most relevant is the alternative assumptions under which they were derived. The DP approach does not need the assumptions that generate invertibility of the variable input demand function. So, e.g., it can allow for unobserved cost shocks to all inputs, unlike our approach, which does not allow such shocks to the price of $m_{i t}$. On the other hand, the DP derivation seems to rely on the linearity of the $\omega_{i t}$ process - in contrast, OP, LP, and our approach can treat the first-order markov process completely non-parametrically. There are other differences between the models. For example, the DP literature can be extended to allow for a fixed effect $\alpha_{i}$ in addition to the $\operatorname{AR}(1)$ process, while generally speaking, this is challenging in our context because it would tend to violate the scalar unobservable assumption. The DP literature also can also potentially allow future values of the intermediate input or investment variable to depend on past $\epsilon_{i t}$ 's, while our approach cannot. On the other hand, as elaborated on in OP, the scalar unobservable assumption of OP/LP and our approach makes it fairly straightforward to extend the methodologies to address endogenous exit (selection) from a sample - this would be considerably harder in the DP context. In summary, both approaches require strong (but different) assumptions. In some cases, a-priori beliefs about a particular production process and/or data considerations may guide choices between the two approaches. In other cases, one may want to try both techniques. Finding that estimates are consistent across multiple techniques with different assumptions is surely more convincing than only using one.

\subsubsection{Units of Measure}

The above discussion has implicitly assumed an "ideal" data scenario where all the variables are measured in physical units that are equivalent across firms. While $l_{i t}$ is often measured in physical units (number of workers, or number of hours), output and inputs such as capital and materials are probably most frequently measured in 
monetary units. When output or an input is measured in monetary values, there are a few things that must be considered to apply the above techniques.

First, assume that firms are price takers in both input and output markets. The first question is whether the prices of equivalent inputs or output vary across firms. ${ }^{11}$ If there is no variation in prices, then the monetary units are comparable across firms and one can apply the above techniques straightforwardly. On the other hand, if the prices of those equivalent inputs (or output) do vary across firms, then the monetary units are not comparable across firms (see, e.g. Klette and Griliches (1996)). In this case, the price variation must be observed for straightforward application of the above techniques. When the price variation is observed, then one can easily divide by prices to obtain measures of physical units and one can apply the above (note that the observed input and output prices need to be included in the intermediate input (or investment) demand function). Note that the observed input prices could also be used as additional instruments for the moment conditions under appropriate assumptions (e.g. that those input prices are exogenous) - see, e.g. Doraszelski and Jaumandreau (2013).

Alternatively, consider a situation where firms face downward sloping output demand curves (and/or upward sloping input supply curves). In this case, one will generally need to assume that firms are all facing identical demand/supply curves - otherwise firms will have different intermediate input (or investment) demand functions (i.e. the scalar unobservable Assumption (4), (4b), or (4c) will be violated). But even if this is true, there is still a problem since firms will generally be operating on different points of those demand and supply curves. Because of the resulting price differences, monetary values of inputs or output will again generally not be comparable across firms. If one observes the price differences of equivalent inputs or outputs, one can again construct measures of equivalent physical units and apply the above. If one does not observe the price differences, then other techniques are required - see Klette and Griliches (1996), De Loecker (2011), and De Loecker and Warzynski (2012) for recent methodological advances for this situation.

\section{Monte-Carlo Experiments}

The discussion above implies that whether OP, LP, or our procedure generates consistent estimates of the production function parameters depends on the underlying DGP. What we instead focus on in our Monte-Carlo experiments is how the various procedures perform under misspecification. Specifically, we consider misspecification where the intermediate input or investment variable is measured with error. In this case, none of the procedures produce consistent estimates, but we illustrate (in at least one setup) that our procedure appears less affected by this misspecification than OP and LP. Note that since the original version of the paper, others have proposed newer methods of explicitly addressing measurement error in inputs - Hu and Huang (2011) propose a method to explicitly deal with such measurement error in these approaches based on observing multiple proxies and deconvolution methods, and Kim, Petrin, and Song (2013) also allow for measurement error in capital.

Our Monte-Carlo setup extends Syverson (2001) and Van Biesebroeck (2007), who consider a simple dynamic model of firm investment and production that is analytically solvable (which considerably eases computation burden and reliability issues for Monte-Carlo purposes). The parameters of the model were chosen to match a couple of key moments in the Chilean data used by LP. The full model setup is described in the Appendix, but the basics are as follows. Productivity follows a first-order AR(1) process. Firms make optimal choices of investment in the capital stock to maximize the expected (discounted) value of future profits, where there are convex capital adjustment costs, and as above, the period $t$ capital stock is determined by investment at $t-1$ (i.e. $\left.K_{i t}=(1-\delta) K_{i t-1}+I_{i t-1}\right)$. Material inputs $m_{i t}$ are chosen at $t$, while labor input $l_{i t}$ is either chosen at $t$ or at $t-b$ (in the latter case, labor is chosen with only knowledge of $\omega_{i t-b}$, not $\omega_{i t}$ ). The production function is assumed Leontief in (and proportional

\footnotetext{
${ }^{11}$ When we describe measures of inputs and outputs as being equivalent across firms, we are ruling out a situation where input or output price differences across firms reflect differences in quality. If one thinks that quality differences are important, monetary measures of input or outputs may be superior to (non-equivalent) physical measures, though as noted below, using monetary measures requires an assumption that firms face the same menu of prices (menu over different quality levels).
} 
to) materials, i.e.

$$
Y_{i t}=\min \left\{\beta_{0} K_{i t}^{\beta_{k}} L_{i t}^{\beta_{l}} \exp \left(\omega_{i t}\right), \beta_{m} M_{i t}\right\} \exp \left(\epsilon_{i t}\right)
$$

where $\beta_{0}=1, \beta_{k}=0.4, \beta_{l}=0.6$, and $\beta_{m}=1$. Given the Leontief first order condition holds, this means that we can proceed using the following "structural value added" (see Gandhi, Navarro and Rivers (2014)) (log) production function

$$
y_{i t}=\beta_{0}+\beta_{k} k_{i t}+\beta_{l} l_{i t}+\omega_{i t}+\epsilon_{i t}
$$

The other free parameters of the model were chosen such that the steady state of the model approximately matches the overall relationship between capital and labor in the Chilean data used by LP. Specifically, in our baseline specifications, $95 \%$ of the variation in capital in the data is across-firm variation, and the $R^{2}$ of a regression of capital on labor is approximately 0.5. Importantly, to match these two "moments" of the Chilean data, we needed to allow unobserved heterogeneity across firms in capital adjustment costs (see the Appendix for details). This violates the scalar unobservable assumption if one is using the investment variable of OP, and highlights the advantages of the LP intermediate input variable approach over the investment variable approach as described earlier - i.e. the intermediate input approach (either with the LP moments or our moments) allows unobserved variation across firms in investment prices or capital adjustment costs. As a result, we primarily compare results using LP with the intermediate input variable, to our approach also using the intermediate input variable.

We consider two base DGPs. The first, DGP1, is favorable to our procedure in that we add aspects to the DGP under which our moments produce consistent estimates but the LP moments do not. First, we assume that firms face different (unobserved to the econometrician) wages, where the wage process for firm $i$ follows an AR(1) process. Second, we assume labor is chosen at time time $t-b$, where $b=0.5$ and where $\omega$ follows an AR(1) process both between $\omega_{i t-1}$ and $\omega_{i t-b}$ and between $\omega_{i t-b}$ and $\omega_{i t}$. Details are in the Appendix. As discussed in Section 3, the reason that our moments produce consistent estimates in this DGP is because we use the conditional (on $l_{i t}$ ) intermediate demand function, while LP does not. ${ }^{12}$

The second DGP (DGP2) is favorable to LP. There is no wage variation across firms, and labor is chosen at $t$ with full information on $\omega_{i t}$, so the model is consistent with the LP moments. We add simple optimization error in labor, where the labor variable is essentially the optimal level of labor plus i.i.d. noise. As noted above, this seems to be the simplest way to overcome the functional dependence issue in a way that is consistent with the LP assumptions (recall that choice of $m_{i t}$ cannot respond to the optimization error in $l_{i t}$, so in this DGP the $m_{i t}$ that is observed needs to be "planned materials", i.e. the $m_{i t}$ chosen prior to the realization of optimization error in $l_{i t}$.) While our procedure also should produce consistent estimates with this DGP, we suspect that our estimates will not be as efficient as LP, since in this case, the LP moments can be seen as a restricted version of our moments (where that additional restriction is valid). In the context of our moments, the additional (valid) restriction is that $\widetilde{\Phi}_{t}$ is linear in $l_{i t}$ (with the coefficient $\beta_{l}$ ).

We also consider a third DGP (DGP3) that is a combination of the above two, i.e. it includes optimization error in $l_{i t}$, serially correlated wage variation across firms, and labor being chosen at time $t-b$. Neither the LP moments nor our moments produce consistent estimates under DGP3. While our procedure produces consistent estimates under DGP1 or DGP2 individually, it does not produce consistent estimates under the combination of them because in DGP2, the $m_{i t}$ that is observed depends on $l_{i t}$ without optimization error. Hence, the inverted

\footnotetext{
${ }^{12}$ Note that along with the assumption that firms are price takers in input and output markets, this particular "proportional in $M_{i t}$ " Leontief specification avoids Gandhi, Navarro, and Rivers (2014) critique of using these techniques with Leontief production functions. More specifically, in this setup, capital and labor will not be idled unless the firm chooses to shutdown (if the scaled price of materials is somehow less than the price of output), and if the firm shuts down they would presumably not be in the data set. Moreover, there is not even this type of optimal shut down in our Monte-carlos, since there is no across firm variation in the price of materials or output. See the appendix for more details.
} 
materials demand function that is used, i.e. a function of the observed $l_{i t}$ with optimization error, is not correct. ${ }^{13,14}$

In each of these 3 DGPs, we start with a base case in which there is no measurement error in the materials variable. ${ }^{15}$ We then add increasing levels of such i.i.d. measurement error. Again, as soon as this measurement error is added, neither LP nor our procedure produce consistent estimates - our goal is to see how sensitive the various procedures are to the additional measurement error. The top third of Table 1 contains results for DGP1 - in the first row, there is 0 measurement error in the material input variable, and in later rows, the level of this measurement error is increased. Measurement error of, e.g., 0.1 indicates that the measurement error is of a magnitude that increases the variance of $m_{i t}$ by $10 \%$.

The first row demonstrates that, as expected, even without measurement error the LP moments do not produce what appear to be consistent estimates. On the other hand, our moment conditions do appear to produce consistent estimates when there is no measurement error. Interestingly, as we change the level of measurement error in $m_{i t}$, the LP estimate of the labor coefficient is considerably more sensitive, ranging from way below the true value of 0.6 with no measurement error, to considerably above the true value when there is lots of such error. Estimates using our moment conditions are again fairly stable, though there seems to be a small positive bias as the measurement error increases from 0 .

The middle third of Table 1 contains results from DGP 2. As noted above, with no measurement error, both our procedure and the LP procedure effectively find the true coefficients on labor and capital. Also as expected, the LP estimates are considerably more precise than those using our moments. However, as measurement error in materials is added, the LP labor coefficient increases and the LP capital coefficients decrease away from the true parameters. The capital parameter using our moments seem to be relatively unaffected by the measurement error in materials, while the labor coefficients do move somewhat. However, generally speaking the LP coefficient estimates seem more affected by the measurement error than do the coefficient estimates based on our moments.

The last third of Table 1 contains results from DGP 3. Again, neither procedure is consistent in this case, even without measurement error in $m_{i t}$. But without the measurement error, the estimates based on our moments are closer to the truth than the LP estimates. Moreover, we again find that our estimates are much less sensitive than the LP estimates to measurement error. For $\beta_{l}$ and $\beta_{k}$, our moments generate estimates that are at most only about $10 \%$ away from the truth. In contrast, the LP estimate of the labor coefficient, for example, varies between 0.47 and 0.68. These results suggesting that our moments are more robust to misspecification than the LP moments seem consistent with results in a working paper version of this paper. There, using the actual Chilean data, we found that while results using our moments were relatively robust across a variety of intermediate input proxies (materials, electricity, fuels), results using the LP moments were more variable.

Lastly, we tried the OP estimator using investment to recover unobserved productivity. As noted previously, given our use of firm-specific capital adjustment costs to reproduce observed patterns in the data, OP will not be consistent under any of these DGPs. In fact, the misspecification due to the firm specific adjustment costs seems to dwarf any misspecification due to measurement error (now in the investment variable). As a result, regardless of the level of that measurement error or the specification, we obtain labor coefficients estimates of approximately 0.82 to 0.87 (Table 2). This again highlights how the general idea of Levinsohn and Petrin to use the intermediate

\footnotetext{
${ }^{13}$ This turns out to not matter in DGP2 because in DGP2, $m_{i t}$ can be written as a function of just $k_{i t}$ and $\omega_{i t}$, and thus including the incorrect $l_{i t}$ is irrelevant. As noted in the appendix, our procedure would produce consistent estimates under a combination of DGP1 and DGP2 if DGP2 were altered such that the $m_{i t}$ that is observed optimally responds to the optimization error in $l_{i t}$. We did not use this alternative DGP2, because under this alternative assumption, LP is not consistent.

${ }^{14}$ For comparison purposes, standard OLS estimates of the production function across these three DGPs produces labor coefficients between 0.85 and 0.95 and capital coefficients between 0.06 and 0.17 .

${ }^{15}$ As pointed out in a prior version of this paper, there is a identification caveat using our suggested moments in all three of these DGPs. More specifically, there is a "global" identification issue in that the moments have expectation zero not only at the true parameters, but also at one other point on the boundary of the parameter space where $\widehat{\beta}_{k}=0, \widehat{\beta}_{l}=\beta_{l}+\beta_{k}$, and the estimated AR(1) coefficient on $\omega$ equals the $\operatorname{AR}(1)$ coefficient on the wage process. One can easily calculate that at these alternative parameter values, the second stage moment equals the innovation in the wage process, which is orthogonal to $k_{i t}$ and $l_{i t-1}$. This "spurious" minimum is a result of labor satisfying a static first order condition, and we suspect it would not occur were labor to have dynamic implications, nor when the alternative moments (29) are assumed. As such, we ignore this spurious minimum in our Monte-Carlos.
} 
input to invert productivity (particularly in the context of our alternative moment conditions) may be preferable to the original OP idea of using investment.

\section{Conclusions}

We argue that some popular production function estimation techniques introduced by Olley and Pakes (1996) and Levinsohn and Petrin (2003) may suffer, at least conceptually, from a functional dependence problem. We propose an alternative, though quite related, estimator that avoids this functional dependence problem. The alternative estimator differs from OP and LP in that it inverts input demand functions that are conditional on choice of labor input (while OP/LP invert unconditional input demand functions). We show how this allows for more general underlying DGPs than does LP, similar to how LP allowed for some more general DGPs than did OP. In comparison to Wooldridge's (2009) stacked version of LP's moments (again based on unconditional input demands), our moments based on conditional input demands are strictly less restrictive. Again, this means that our estimator is strictly more general than that proposed by Wooldridge, e.g. allowing unobserved heterogeneity in labor input prices across firms, allowing labor to have dynamic implications, and allowing labor to possibly be chosen prior to materials with a different information set. As might be expected, this generality and robustness in comparison to Wooldridge and LP does come at an efficiency cost, i.e. when the assumptions behind the unconditional input demand function approach are in fact correct, estimates based on our conditional approach are less efficient. These findings are born out by Monte-Carlo experiments that compare performance of the estimators under various DGPs that are sometimes consistent with the assumptions behind the estimators and sometimes not.

\section{References}

Ackerberg, D., Benkard, L., Berry, S., and Pakes, A. (2007) "Econometric Tools for Analyzing Market Outcomes", Handbook of Econometrics. Amsterdam: North-Holland.

Ackerberg, D. Chen, X., Hahn, J., and Liao, Z. (2014). "Asymptotic Efficiency of Semiparametric Two-step GMM," Review of Economic Studies, 81(3): 919-943.

Ai, C. and Chen, X. (2007) "The Semiparametric Efficiency Bound for Models of Sequential Moment Restrictions Containing Unknown Functions," Journal of Econometrics, 170: 442-457.

Ai, C. and Chen, X. (2003) "Efficient Estimation of Models with Conditional Moment Restrictions Containing Unknown Functions," Econometrica, 71(6): 1795-1843.

Alvarez, R. and Lopez, R. (2005) "Exporting and Performance: Evidence from Chilean Plants" Canadian Journal of Economics, 38(4): 1384-1400.

Anderson T.W. and C. Hsiao (1982) "Formulation and Estimation of Dynamic Models using Panel Data" Journal of Econometrics 18: 47-82.

Arellano, M. and S. Bond (1991) "Some Tests of Specification for Panel Data: Monte Carlo Evidence and an Application to Employment Equations" The Review of Economic Studies 58: 277-297.

Arellano, M. and Bover, O. (1995) "Another Look at the Instrumental Variable Estimation of Error Components Models" Journal of Econometrics, 68: 29-51

Bajari, P. and Benkard, L. (2005) "Demand Estimation with Heterogeneous Consumers and Unobserved Product Characteristics: A Hedonic Approach", Journal of Political Economy, 113(6): 1239-1276.

Basu, S. and J. Fernald (1997) "Returns to Scale in U.S. Production: Estimates and Implications" Journal of Political Economy 105: 249-283.

Basu, S. (1999) - Slides for the discussion of Levinsohn and Petrin (1999) 
Blalock, G. and Gertler, P. (2004) "Learning from Exporting: Revisited in a Less Developed Setting", Journal of Development Economics, 75(2): 397-416.

Blundell, R. and Bond, S. (1998) "Initial Conditions and Moment Restrictions in Dynamic Panel Data Models" Journal of Econometrics 87: 115-143.

Blundell, R. and Bond, S. (2000) "GMM estimation with persistent panel data: an application to production functions", Econometric Reviews 19: 321-340

Bond, S. and Söderbom, M. (2005) "Adjustment Costs and the Identification of Cobb-Douglas Production Functions," The Institute for Fiscal Studies, Working Paper Series No. 05/04.

Bruno, M. (1978). "Duality, Intermediate Inputs, and Value-Added." in Production Economics: A Dual Approach to Theory and Applications, vol. 2., ed. M. Fuss and D. McFadden, Chapter 1. Amsterdam: North-Holland.

Buettner, T. (2005) "Productivity Dynamics when Labor is a State Variable", mimeo, LSE

Chamberlain, G. (1982) "Multivariate Regression Models for Panel Data", Journal of Econometrics 18: 5-46.

Chen, X., Hahn, J. and Liao, Z. (2014) "Semiparametric Two-Step GMM Estimation with Weakly Dependent Data," mimeo, UCLA.

Chen, X. Hahn, J. Liao, Z. and Ridder, G. (2013) "Asymptotic Properties of Nonparametric Two-Step Sieve Estimates," mimeo, UCLA.

Christensen, L.R. and Greene, W.H. (1976). "Economies of Scale in U.S. Electric Power Generation," Journal of Political Economy, 84(4): 655-676.

Christensen, L.R., Jorgenson, D.W. and Lau, L.J. (1973) "Transcendental Logarithmic Production Frontiers," Review of Economics and Statistics, 55(1): 28-45.

Criscuolo, C. and Martin, R. (2009) "Multinationals and U.S. Productivity Leadership: Evidence from Great Britain", Review of Economics and Statistics, 91(2): 263-281.

De Loecker, J. (2011) "Product Differentiation, Multi-Product Firms and Estimating the Impact of Trade Liberalization on Productivity," Econometrica, 79(5): 1407-1451.

De Loecker, J. and Warzynski, F. (2012) "Markups and Firm-level Export Status," American Economic Review, 102 (6): 2437-2471.

Diewert, W. Erwin. (1978). "Hick's Aggregation Theorem and the Existence of a Real Value Added Function." In Production Economics: A Dual Approach to Theory and Practice, vol. 2, ed. M. Fuss and D. McFadden, Chapter 1. Amsterdam: North-Holland.

Dollar, D., Hallward-Dreimeier, M. and Mengistae, T. (2005) "Investment Climate and Firm Performance in Developing Countries", Economic Development and Cultural Change: 54(1): 1-31.

Doraszelski, U. and Jaumandreu, J. (2013) "R\&D and Productivity: Estimating Endogenous Productivity," Review of Economic Studies, 80: 1338-1383.

Fernandes, A. (2007) "Trade Policy, Trade Volumes and Plant-Level Productivity in Colombian Manufacturing Industries," Journal of International Economics, 71(1): 52-71.

Gandhi, A. Navarro, S., and Rivers, D. (2014) "On the Identification of Production Functions: How Heterogeneous is Productivity," mimeo, U-Wisconsin-Madison.

Griliches, Z. and Hausman, J. (1986) "Errors in Variables in Panel Data" Journal of Econometrics, 31: 93-118

Griliches, Z. and J.Mairesse. (1998) "Production Functions: The Search for Identification." In: Econometrics and Economic Theory in the Twentieth Century: The Ragnar Frisch Centennial Symposium, ed. S. Strøm. Cambridge, UK: Cambridge University Press. 
Hall, R.E. (1988). "The Relation Between Price and Marginal Cost in U.S. Industry," Journal of Political Economy 96: 921-947.

Hardle, W., M. Muller, S. Sperlich and A. Werwatz, (2004) Nonparametric and Semiparametric Models. Berlin: Springer.

Hoch, I. (1955) "Estimation of production function parameters and testing for efficiency," Econometrica 23: 325326.

Hoch, I. (1958) "Simultaneous Equation Bias in the Context of the Cobb-Douglas Production Function", Econometrica 26: 566-578.

Hoch, I.(1962) "Estimation of Production Parameters Combining Time-Series and Cross-Section Data" Econometrica 30: $34-53$.

Huang, G. and Hu, Y. (2011). "Estimating Production Functions with Robustness Against Errors in the Proxy Variables," mimeo, Johns Hopkins University.

Ivanenko, V. (2003) "Credit Rationing and the Shadow Value of Money in Trade", mimeo, U Western Ontario.

Kasahara, H. and Rodrique, J. (2008) "Does the Use of Imported Intermediates Increase Productivity? Plant Level Evidence", Journal of Development Economics, 87(1): 106-118.

Kim, Kyoo il, Petrin, A. and Song, S. (2013) "Estimating Production Functions with Control Functions when Capital is Measured with Error," mimeo, University of Chicago.

Klein, L.R. (1953) A Textbook of Econometrics. Prentice-Hall, NJ.

Klette, T. J. and Griliches, Z. (1996) "The Inconsistency of Common Scale Estimators when Output Prices are Unobserved and Endogenous," Journal of Applied Econometrics, 11 (4): 343-61.

Levinsohn, J. and Petrin, A. (2003) "Estimating Production Functions Using Inputs to Control for Unobservables," Review of Economic Studies 70: 317-342

Marschak, J. and Andrews, Jr., William H. (1944) "Random Simultaneous Equations and the Theory of Production," Econometrica 12: 143-205.

Matzkin, R.(2003) "Nonparametric Estimation of Non-Additive Random Functions," Econometrica 71: 1339-1375.

McElroy, M.B. (1987) "Additive General Error Models for Production, Cost, and Derived Demand or Share Systems," Journal of Political Economy, 95(4): 737-757.

Mundlak, Y.(1961). "Empirical Production Function Free of Management Bias." Journal of Farm Economics: 44-56.

Mundlak, Y. (1963). "Estimation of Production and Behavioral Functions from a Combination of Cross-Section and Time-Series Data," in C. Christ et. al., eds., Measurement in Economics. Stanford, CA: Stanford University Press, 138-166.

Mundlak, Y. (1996). "Production Function Estimation: Reviving the Primal," Econometrica, 64(2): 431-438.

Mundlak, Y. and I. Hoch. (1965). "Consequences of Alternative Specifications in Estimation of Cobb-Douglas Production Functions," Econometrica 33: 814-828.

Nerlove, M. (1963) "Returns to Scale in Electricity Supply," in Christ et. al., eds., Measurement in Economics. Stanford: Stanford University Press, 167-200.

Olley, S. and Pakes, A.(1996) "The Dynamics of Productivity in the Telecommunications Equipment Industry" Econometrica 64:1263-1295

Ozler, S. and Yilmaz, K. (2001) "Productivity Response to Reduction in Trade Barriers: Evidence from Turkish Manufacturing Plants", Review of World Economics, 145(2): 339-360. 
Pagan, A. (1986) "Two Stage and Related Estimators and their Applications" Review of Economic Studies 517-538

Pakes, A. and Olley, S. (1995). "A Limit Theorem for a Smooth Class of Semiparametric Estimators," Journal of Econometrics 65: 295-332.

Panzar, J. (1989). "Technological Determinants of Firm and Industry Structure," in R. Schmalensee and R. Willig, eds., Handbook of Industrial Organization, vol. 1. Amsterdam: North-Holland, 3-59.

Pavcnik, N. (2002) "Trade Liberalization Exit and Productivity Improvements: Evidence from Chilean Plants," Review of Economic Studies 69: 245-276.

Robinson, P.M. (1988) "Root-N-Consistent Semiparametric Regression," Econometrica 56: 931-954.

Sivadasan, J. (2009) "Barriers to Competition and Productivity: Evidence from India," B.E. Journal of Economic Analysis \& Policy: 9(1), Article 42.

Slade, M. (1989) "Modeling Stochastic and Cyclical Components of Technical Change: An Application of the Kalman Filter," Journal of Econometrics, 41: 363-83.

Solow, R.M. (1957) "Technical Change and the Aggregate Production Function," Review of Economics and Statistics, 39: 312-320.

Syverson, C. (2001) Market Structuure and Productivity. Ph. D. dissertation, University of Maryland.

Topalova, P. and Khadewal, A. (2011) "Trade Liberalization and Firm Productivity: The Case of India", Review of Economics and Statistics, 93(3): 995-1009.

Van Biesebroeck, J. (2003) "Productivity Dynamics with Technology Choice: An Application to Automobile Assembly", Review of Economic Studies 70(1): 167-198.

Van Biesebroeck, J. (2007) "Robustness of productivity estimates," The Journal of Industrial Economics , 55: $529-569$

Wooldridge (2009) "On Estimating Firm-Level Production Functions Using Proxy Variables to Control for Unobservables," Economics Letters, 104(3): 112-114. 


\section{Monte-Carlo Setup}

We now describe our Monte-Carlo setup. We consider a panel of 1000 firms over 10 time periods. The parameters of the model are chosen such that the capital and labor series approximate key aspects of the Chilean data used in LP. Specifically, in our baseline specifications, $95 \%$ of the variation in capital in the data is across-firm variation (vs within-firm variation), and the $\mathrm{R}^{2}$ of a regression of capital on labor is approximately 0.5 .

\subsection{Production Function and Productivity Shocks}

We use a production function that is Leontief in the material input, i.e.

$$
Y_{i t}=\min \left\{\beta_{0} K_{i t}^{\beta_{k}} L_{i t}^{\beta_{l}} e^{\omega_{i t}}, \beta_{m} M_{i t}\right\} e^{\epsilon_{i t}}
$$

where $\beta_{0}=1, \beta_{k}=0.4, \beta_{l}=0.6$, and $\beta_{m}=1$. Note that in this specification, material input is proportional to output. This is perhaps the most natural Leontief specification, corresponding to a situation where a specific, fixed, amount of the material input needs to be used to produce one unit of output (e.g. some specific amount of metal required to produce a can to hold food, some amount of wood required to produce an item). $\epsilon_{i t}$ is mean 0 measurement error with standard deviation 0.1 that is distributed iid over firms and time. The productivity shock $\omega_{i t}$ follows the following $\mathrm{AR}(1)$ process

$$
\omega_{i t}=\rho \omega_{i t-1}+\xi_{i t}
$$

where $\rho=0.7$. The variances of the normally distributed innovation $\xi_{i t}\left(\sigma_{\xi}^{2}\right)$ and the initial value $\omega_{i 0}\left(\sigma_{\omega_{i 0}}^{2}\right)$ are set such that the standard deviation of $\omega_{i t}$ is constant over time and equal to 0.3 .

\subsection{Choice of Labor and Material Inputs}

As described in Section 5, our specifications differ in how they model the DGP generating the labor (and material) inputs. In all specifications, we assume that labor and materials are not dynamic inputs, i.e. a firm's choice of $L_{i t}$ and $M_{i t}$ has no implication for profitability after period $t$. As noted in the main text, our procedure is consistent if labor has dynamic implications. We did not allow this in our Monte-Carlo's - for speed and reliability purposes we wanted a model where the firms dynamic programming problem can be solved analytically. When the only dynamic input is capital, we can extend Syverson (2001) and Van Biesebroeck (2007) to obtain a model for which the optimal investment level can be solved analytically. An alternative would be to follow Bond and Söderbom (2005), allow dynamics (e.g. adjustment costs) in both capital and labor and solve the model numerically.

We consider two main data generating processes. DGP1 allows there to be firm specific (unobserved to the econometrician) wage shocks, and also allows $L_{i t}$ to be chosen prior to period $t$, i.e. without full knowledge of $\omega_{i t}$. DGP1 is favorable to our procedure in that our conditional input demand approach produces consistent estimates, but the LP unconditional approach does not. In contrast, DGP2 does not allow firm specific wage shocks, and $L_{i t}$ is chosen with knowledge of $\omega_{i t}$. However, DGP2 does add optimization error to $L_{i t}$ (while there is no optimization error in $M_{i t}$ ). DGP2 is favorable to LP, as the LP procedure produces consistent estimates. While our procedure also produces consistent estimates, we expect them to have higher standard errors than those generated by LP.

More specifically, DGP1 first allows labor to be chosen at time period $t-b$ (we set $b=0.5$ ). We can think of decomposing the AR(1) process (36) into two subprocesses. First $\omega_{i t-1}$ evolves to $\omega_{i t-b}$, at which point in time the firm chooses labor input (as a function of $\omega_{i t-b}$ ). Then, after $L_{i t}$ is chosen, $\omega_{i t-b}$ evolves to $\omega_{i t}$. We use the following model of the evolution of $\omega$ between sub-periods:

$$
\begin{aligned}
\omega_{i t-b} & =\rho^{1-b} \omega_{i t-1}+\xi_{i t}^{A} \\
\omega_{i t} & =\rho^{b} \omega_{i t-b}+\xi_{i t}^{B}
\end{aligned}
$$

Thus, when $b>0$, firms have less than perfect information about $\omega_{i t}$ when chosing $L_{i t}$, and when $b$ increases, this information decreases. Note that this is consistent with the $\operatorname{AR}(1)$ coefficient in (36) since $\rho^{1-b} \rho^{b}=\rho$. Also for consistency with (36), we impose that $\operatorname{Var}\left(\rho^{b} \xi_{i t}^{A}+\xi_{i t}^{B}\right)=\operatorname{Var}\left(\xi_{i t}\right)$ and that the variance of $\xi_{i t}^{A}$ is such that the variance of $\omega_{i t-b}$ is constant over time. This defines $\operatorname{Var}\left(\xi_{i t}^{A}\right) \equiv \sigma_{\xi^{A}}^{2}$ and $\operatorname{Var}\left(\xi_{i t}^{B}\right) \equiv \sigma_{\xi^{B}}^{2}$, and intuitively implies that $\sigma_{\xi^{A}}^{2}$ is decreasing in $b$ and $\sigma_{\xi^{B}}^{2}$ is increasing in $b$. 
In DGP1 firms also face different wages where the (ln) wage process for firm $i$ follows an AR(1) process

$$
\ln \left(W_{i t}\right)=0.3 \ln \left(W_{i t-1}\right)+\xi_{i t}^{W}
$$

where the variances of the normally distributed innovation $\xi_{i t}^{W}\left(\sigma_{\xi^{W}}^{2}\right)$ and the initial value $\ln \left(W_{i 0}\right)$ are set such that the standard deviation of $\ln \left(W_{i t}\right)$ is constant over time and equal to 0.1. Relative to a baseline in which all firms face the mean log wage in every period (1), this wage variation increases the within-firm, across-time, standard deviation of $\ln \left(L_{i t}\right)$ by about $10 \%$.

Given DGP1, firms optimally choose $L_{i t}$ to maximize expected profits by setting (with the difference between the price of output and the price of the material input normalized to 1 ):

$$
L_{i t}=\beta_{0}^{\frac{1}{1-\beta_{l}}} \beta_{l}^{\frac{1}{1-\beta_{l}}} W_{i t}^{\frac{-1}{1-\beta_{l}}} K_{i t}^{\frac{\beta_{k}}{1-\beta_{l}}} e^{\left(\frac{1}{1-\beta_{l}}\right)\left(\rho^{b} \omega_{i t-b}+\frac{1}{2} \sigma_{\xi^{B}}^{2}\right)}
$$

Note that DGP1 is not subject to the critique of Gandhi, Navarro and Rivers (2014) of Leontief production functions (as noted in footnote 12 of the main paper). They discuss how in the context of a Leontief production function, e.g.

$$
Y_{i t}=\min \left\{\beta_{0} K_{i t}^{\beta_{k}} L_{i t}^{\beta_{l}} e^{\omega_{i t}}, m\left(M_{i t}\right)\right\} e^{\epsilon_{i t}}
$$

it is possible that the Leontief "first-order condition"

$$
m\left(M_{i t}\right)=\beta_{0} K_{i t}^{\beta_{k}} L_{i t}^{\beta_{l}} e^{\omega_{i t}}
$$

might not hold. The intuition for their argument is as follows. Suppose $K_{i t}$ and $L_{i t}$ are chosen prior to $M_{i t}$ being chosen. Suppose that between these two points in time, the price of $M_{i t}$ rises precipitously (and unexpectedly), such that the revenue from selling output doesn't even cover the cost of the material input required to produce that output (i.e. ignoring the "sunk" costs of already purchased $K_{i t}$ and $L_{i t}$ ). In this case, firms would not generally choose $M_{i t}$ to satisfy (39), and thus the data could contain points where

$$
Y_{i t} \neq \beta_{0} K_{i t}^{\beta_{k}} L_{i t}^{\beta_{l}} e^{\omega_{i t}+\epsilon_{i t}}
$$

invalidating all the above procedures. However, in our Monte-Carlo setup where output is proportional to material input (i.e. $m\left(M_{i t}\right)=\beta_{m} M_{i t}$, which could be the most plausible Leontief specification for many material inputs), this is not a problem. This is because firms will either satisfy (39) or produce 0 output (the latter if the price of $M_{i t}$ unexpectedly rises enough after the other inputs are chosen), and if they produce zero, they will presumably not be in the dataset of firms that are operating and thus not be a problem for estimation. Moreover, in our Monte-Carlos the price of material inputs (and output) are constant across firms anyway, so firms never want to let capital and labor go unutilized and produce $0 .{ }^{16}$

In contrast to DGP1, in DGP2 firms face identical wages and $L_{i t}$ is chosen at period $t$ (i.e. $b=0$ ). These changes are needed to make the model consistent with the assumptions of LP. Moreover, we need something to break the functional dependence issue, so we add optimization error to $L_{i t}$. More specifically, let $L_{i t}^{\text {err }}$ be the firms choice of labor including optimization error. We assume that

$$
L_{i t}^{e r r}=L_{i t} e^{\xi_{i t}^{l}}
$$

where $\xi_{i t}^{l} \sim N\left(0, \sigma_{\xi^{l}}^{2}\right)$ is optimization error that is distributed iid over firms and time (and independent of everything else in the model). We set $\sigma_{\xi^{l}}=0.37$, which also increases the within-firm, across-time, standard deviation of $\ln \left(L_{i t}\right)$ by about $10 \%$.

Constructing this DGP such that LP produces consistent estimates is somewhat subtle. If the firm observes $L_{i t}^{e r r}$ prior to choosing the material input, they will typically choose

$$
M_{i t}^{e r r}=\beta_{0} K_{i t}^{\beta_{k}} L_{i t}^{e r r^{\beta_{l}}} e^{\omega_{i t}}
$$

\footnotetext{
${ }^{16}$ Note that in this example, a fully non-parametric first stage regression of $Y_{i t}$ on $K_{i t}$, $L_{i t}$, and $M_{i t}$ to recover $\epsilon_{i t}$ is not strictly needed given the assumed Leontief functional form. For example, one could alternatively regress $Y_{i t}$ on just $M_{i t}$ to recover $\epsilon_{i t}$, or similarly, use the Leontief FOC directly. In our Monte-Carlos, we do the fully general first stage (perhaps sacrificing some efficiency) because that corresponds to our more general procedure.
} 
This is a problem if the econometrician observes $M_{i t}^{e r r}$ as the material input measure, as this would violate the scalar unobservable assumption of LP (Assumption (4b)). So instead we assume that the econometrician observes $M_{i t}$ instead of $M_{i t}^{e r r}$, where

$$
M_{i t}=\beta_{0} K_{i t}^{\beta_{k}} L_{i t}^{\beta_{l}} e^{\omega_{i t}}
$$

One interpretation of this is that, after observing $\omega_{i t}$, firms commit to "planned" optimal $L_{i t}$ and $M_{i t}$. However, workers are randomly sick, and when more workers are sick less materials are used, so that the firm ends up using $L_{i t}^{e r r}$ and $M_{i t}^{e r r}$ and producing ${ }^{17}$

$$
Y_{i t}=\beta_{0} K_{i t}^{\beta_{k}} L_{i t}^{e r r^{\beta_{l}}} e^{\omega_{i t}} e^{\epsilon_{i t}}
$$

What the econometrician is assumed to observe are $Y_{i t}, K_{i t}, L_{i t}^{e r r}$, and $M_{i t}$. The interpretation of observing $M_{i t}$ is that what is reported in the data is planned (or ordered) materials, i.e. prior to the realization of sick days and $L_{i t}^{e r r}$.

In DGP2, both LP and our procedure produce consistent estimates. While we do not present results for this, one can consider an alternative DGP2, call it DGP2b, where $M_{i t}^{\text {err }}$ is observed instead of $M_{i t}$. In DGP2b, LP does not produce consistent estimates, but our suggested procedure does (since conditional on $L_{i t}^{e r r}$, the scalar unobservable assumption is not violated). We also consider the combination of DGP1 and DGP2, i.e. a DGP that includes across firm wage variation, labor chosen at $t-b$, and optimization error in $L_{i t}$. In this setup, neither LP nor our procedure produce consistent estimates (see the main text for a discussion of why our procedure does not produce consistent estimates under DGP1 + DGP2, even though it provides consistent estimates under DGP1 or DGP2 separately). While we again do not present results, our procedure would produce consistent estimates under DGP1 + DGP2b, while LP would not.

For all three DGPs (DGP1, DGP2, and DGP3 = DGP1 + DGP2), we additionally add various levels of measurement error to the observed material input. As noted in the main text, this renders both LP and our approach inconsistent. However, we find that estimation based on the LP moments appears to be more sensitive to this measurement error than does estimation based on our moments.

\subsection{Investment Choice and Steady State}

In contrast to non-dynamic labor and material inputs, we assume that capital is a dynamic input. Specifically, capital is accumulated through investment according to

$$
K_{i t}=(1-\delta) K_{i t-1}+I_{i t-1}
$$

where $(1-\delta)=0.8$, and investment is subject to convex adjustment costs given by

$$
c_{i}\left(I_{i t}\right)=\frac{\phi_{i}}{2} I_{i t}^{2}
$$

where $1 / \phi_{i}$ is distributed lognormally across firms (but constant over time) with standard deviation 0.6. While the existence of this unobserved heterogeneity in adjustment costs is consistent with the LP procedure and our procedure, it is not consistent with the scalar unobservable assumption in the OP context. This renders OP estimates inconsistent in all our Monte-Carlos. We included it because without this heterogeneity it was extremely hard (in steady state, see below) to match the within-firm vs across-firm variation in capital and the correlation between capital and labor of the Chilean datasets as discussed above. ${ }^{18}$

As described Syverson (2001) and Van Biesebroeck (2007), under the assumption of constant returns to scale, a pared-down version of the above can be solved analytically using Euler equation techniques. We have verified that their analytic result can be generalized to allow for the extensions in the above model, i.e. persistent across firm

\footnotetext{
${ }^{17}$ In theory, the Leontief critique of GNR could apply here. For example, if between the point in time in which $L_{i t}$ is chosen and $L_{i t}^{e r r}$ is realized, there is a large enough unexpected positive shock to the price of the material input, it is possible that if $L_{i t}^{e r r}>L_{i t}$ the firm would not want to increase material input levels over $M_{i t}$, i.e. the unexpected supply of labor would optimally go unutilized. However, in our setup, there are no such shocks to the price of material input (or output), so everything is internally consistent. Note also that when the econometrician observes planned materials, as in this DGP, one cannot simply estimate the model directly off the Leontief first order condition.

${ }^{18}$ Without heterogeneity in $\phi_{i}$, the only way our DGPs can match $95 \%$ of the variation in $K_{i t}$ being "across-firm variation" is by setting the persistence of the productivity process $\omega_{i t}$ extremely high. But when this persistence is extremely high, it implies a very high correlation between $K_{i t}$ and $L_{i t}$ which contradicts the $\mathrm{R}^{2}$ of a fixed effects regression of $L_{i t}$ on $K_{i t}$ of only about $50 \%$ in the Chilean data, unless one includes unrealistic amounts of optimization error or wage variation.
} 
variation in wages, labor being chosen at $t-b$, and optimization error in $L_{i t}$. Specifically, an Euler equation approach implies the following optimal investment rule (where $\beta$ is the discount factor, set to 0.95 in our Monte-Carlos):

$$
\begin{aligned}
I_{i t}= & \frac{\beta}{\phi_{i}} \sum_{\tau=0}^{\infty}(\beta(1-\delta))^{\tau}\left(\frac{\beta_{k}}{1-\beta_{l}}\right) \beta_{0}^{\frac{1}{1-\beta_{l}}}\left[\beta_{l}^{\frac{\beta_{l}}{1-\beta_{l}}} e^{\frac{1}{2} \beta_{l}^{2} \sigma_{\xi^{l}}^{2}}-\beta_{l}^{\frac{1}{1-\beta_{l}}} e^{\left.\frac{1}{2} \sigma_{\xi^{l}}^{2}\right]}\right. \\
& e^{\left[\left(\frac{1}{1-\beta_{l}}\right) \rho^{\tau+1} \omega_{i t}+\frac{-\beta_{l}}{1-\beta_{l}} \rho_{W}^{\tau+1} \ln \left(W_{i t}\right)+\frac{1}{2}\left(\frac{-\beta_{l}}{1-\beta_{l}}\right)^{2} \sigma_{\xi^{W}}^{2} \sum_{s=0}^{\tau} \rho_{W}^{2(\tau-s)}+\frac{1}{2}\left(\frac{1}{1-\beta_{l}}\right)^{2} \rho_{b}^{2}\left(\rho^{2 \tau} \sigma_{\xi^{a}}^{2}+\sum_{s=1}^{\tau} \rho^{2(\tau-s)} \sigma_{\xi}^{2}\right)+\left(\frac{1}{1-\beta_{l}}\right)\left(\frac{1}{2} \sigma_{\xi^{b}}^{2}\right)\right]}
\end{aligned}
$$

Note that because of the constant returns to scale, optimal investment does not depend on current capital stock the only firm specific variables that it depends on are $\omega_{i t}, \phi_{i}$, and $\ln \left(W_{i t}\right)$ (the latter because it is serially correlated over time).

For our experiments, we did not want our results to depend on an arbitrary, exogenous, initial distribution of capital stock across firms (one would also need to somehow specify correlations between $K_{i 0}$ and $\left(\omega_{i 0}, \phi_{i}, \ln \left(W_{i 0}\right)\right)$. Instead, we decided to take data from the steady state distribution of firms implied by our model. To do this, we simply started all firms with $K_{i 0}=0$, and simulated firms into the future. Our 10 periods of data are taken from a point in time where the impact of the initial values appeared minimal, i.e. the steady state.

\subsection{Estimation}

We estimate the following Leontief-derived value-added production function

$$
Y_{i t}=\beta_{0} K_{i t}^{\beta_{k}} L_{i t}^{\beta_{l}} e^{\omega_{i t}} e^{\epsilon_{i t}}
$$

(or in DGP2 the same with $L_{i t}$ replaced with $L_{i t}^{e r r}$ ). When we use our procedure or LP, we use $M_{i t}$ to invert the productivity shock, and when we run OP, we use $I_{i t}{ }^{19}$.

For our procedure, our first stage is simply OLS of $y_{i t}$ on $k_{i t}, l_{i t}$, and $m_{i t}$. More generally, one would want higher order polynomials in the explanatory variables, but because in our setup, optimal $m_{i t}$ is actually a linear function of $k_{i t}, l_{i t}$, and $\omega_{i t}$, we simply used a first order polynomial. In the second stage, our estimation is based on the following moments (where $\widetilde{\beta}_{0}=\ln \left(\beta_{0}\right)$ ) :

$$
E\left[\left(y_{i t}-\widetilde{\beta}_{0}-\beta_{k} k_{i t}-\beta_{l} l_{i t}-\rho \cdot\left(\widetilde{\Phi}_{t-1}\left(k_{i t-1}, l_{i t-1}, m_{i t-1}\right)-\widetilde{\beta}_{0}-\beta_{k} k_{i t-1}-\beta_{l} l_{i t-1}\right)\right) \otimes\left(\begin{array}{cc}
1 & \\
k_{i t} & \\
l_{i t-1} & \widetilde{\Phi}_{t-1}\left(k_{i t-1}, l_{i t-1}, m_{i t-1}\right)
\end{array}\right)\right]=0
$$

However, to reduce the dimension of the non-linear search (and presumably increase speed and reliability for our experiments), we don't use these moments directly. Instead we "concentrate-out" two of the parameters $\widetilde{\beta}_{0}$ and $\rho$. More specifically, for a hypothetical guess of the parameters $\beta_{k}$ and $\beta_{l}$, we construct

$$
\widetilde{\beta}_{0}+\omega_{i t}\left(\beta_{k}, \beta_{l}\right)=\widehat{\widetilde{\Phi}}_{t}\left(k_{i t}, l_{i t}, m_{i t}\right)-\beta_{k} k_{i t}-\beta_{l} l_{i t}
$$

where, again, $\widehat{\widetilde{\Phi}}_{t}\left(k_{i t}, l_{i t}, m_{i t}\right)$ is the predicted value from the first stage. We then regress $\widetilde{\beta}_{0}+\omega_{i t}\left(\beta_{k}, \beta_{l}\right)$ on $\widetilde{\beta}_{0}+\omega_{i t-1}\left(\beta_{k}, \beta_{l}\right)$, noting that the residuals of this regression are the implied values of the innovations in omega, i.e. $\widehat{\xi}_{i t}\left(\beta_{k}, \beta_{l}\right)$. Note that this regression implicitly makes these innovations mean zero and uncorrelated with $\widehat{\omega_{i t-1}}\left(\beta_{k}, \beta_{l}\right)$, so it is similar to enforcing the first and fourth moments in (40). While generally one might want to do this regression using higher order polynomials in the explanatory variable, we again simply do it linearly, since our true DGPs use a linear $\operatorname{AR}(1)$ process for $\omega_{i t}$. We then search over $\beta_{k}$ and $\beta_{l}$ space to find $\widehat{\beta}_{k}$ and $\widehat{\beta}_{l}$ that minimize the following moment conditions

$$
E\left[\widehat{\xi}_{i t}\left(\beta_{k}, \beta_{l}\right) \otimes\left(\begin{array}{c}
k_{i t} \\
l_{i t-1}
\end{array}\right)\right]=0
$$

\footnotetext{
${ }^{19}$ As noted, one could also use our moments along with $I_{i t}$ as the variable to recover the productivity shock, but because of the unobserved heterogeneity in $\phi_{i}$ in our Monte-Carlo setup, this would, like OP, not generate consistent estimates.
} 
Our estimate of $\rho$ is given by the slope coefficient of the regression of $\widetilde{\beta_{0}+\omega_{i t}}\left(\beta_{k}, \beta_{l}\right)$ on $\widetilde{\beta}_{0}+\widehat{\omega_{i t-1}}\left(\beta_{k}, \beta_{l}\right)$ evaluated at $\widehat{\beta}_{k}$ and $\widehat{\beta}_{l}$.

For our LP estimates, we use the canned STATA routine (Levinsohn, Petrin, and Poi (2004)), edited to again use linear approximations (since according to the DGP, the true functions were in fact linear). Our results were very similar when we used the default routine based on cubic approximations.. For our OP results, we also use the canned LP STATA routine modified to use the investment variable, but here we use the default cubic approximation, since the dynamic investment function is not linear. 